\title{
XXIV.
}

Aus dem Pathologischen Laboratorium der Universität Amsterdam (Direktor: Prof. Dr. P. Ruitinga).

\section{Ueber Erythrophlein.}

I. Mitteilung:

Die Wirkung des Erythrophleins auf das isollerte Froschherz.

\author{
Von \\ Dr. J. B. Polak und A. J. L. Terwen, \\ Assistentoo. \\ (Hierzu Tafelo XIIV-XXVII)
}

In einer Mitteilung von Ginzberg und Hohlberg (1) über die physiologische Wertbestimmung von Herzmitteln wird von ihnen. das Erythrophlein gerühmt wegen seiner Reinheit und seiner konstanten Wirksamkeit. Sie meinen deshalb die Verbindung als die am meisten geeignete Titersubstanz für diese Wertbestimmung betrachten zu müssen. Der Besitz eines Praparates von so zuverlässiger Reinheit und Wirksamkeit erschien uns auch für die Klinik grossen Nutzen abwerten zu können. Wir entschlossen uns deshalb 7.4 einer möglichst vollständigen pharmakodynamischen Untersuchung der Substanz in der Absicht, zu einer versuchsweise angestellten klinischen Verwendung uberzugehen, wemn die Resultate im Laboratorium einen derartigen Versuch reehtfertigen würden. Wir liabeu unsere Experimento angefangen am isolierten Froschherzell, das wir der Wirkung des Erythrophleinum sulfuricum von Mlerck aussetzten.

Vorher benutzte man nur das salzsaure Salz. Dieses ist jedoch eine amorphe, wasseranziehende Verbindung und deshalb weniger zuverlassig als das kristallinische nicht hygroskopische Sulfat.

Das Erythrophlein ist ein Alkaloid, das sich findet in der Rinde ron Erythrophleum guinense, einem prachtvollen Baum, der zur Familie der Caesalpineën gehört. Er wächst in West-Afrika und wird mit vielfachen Namen bezeichnet (Red-water-tree der Englander). In der ersten Beschreibung oun Guillemin, Perrottet und Richard(2) heisst er Fillaea suaveolens. Aus der Rinde (Sassy-Rinde oder Tali, englisch: casca- oder cassabark, französisch: Ecorce de Mancone des Portugais odèr Bourane de Floups) bereiten dic Eingeborenen einen Extrakt, den sie als Pfeilgift und zum Erhalten eines Gottesurteils verwenden. Gallois und Hardy (3) haben zuerst aus der Rinde dise Substanz isoliert und auf die digitalisähnliche Wirkung hingewiesen. Weiter ist das Erythrophlein studiert worden von Brunton und Pyre (4) sowie ron Sée und Bochefontaine (5), die beim Hunde Blutdrucksteigerung, Frequenzabnahme, später vermutlich Herzblock feststellten und im letzten Stadium 
eine Frequenzzunahne, die von Vagusreizung nicht beeinflusst wurde (Vorhofsflimmern?).

Auch hat man versucht das Mittel als Anästhetikum zu verwenden (6). Im Konjunktivalsack gibt es jedoch eine starke Entzündung der Kornea, die sich nachher trübt, während die subkutane Anwendıng sich wegen der Schmerzen nach der Einspritzung und wegen der resorptiven Vergiftungserscheinungen verbietet.

Als Herzmittel ist das Erythrophlein klinisch untersucht worden ron Hermann (7), Sée (8) und Drummond (9) mit geringem Erfolg. Sie sahen nach kleinon Gaben schon ziemlich schwere Vergiftungssymptome, eine Folge ron der Unreinheit ihrer Präparate. Später, bei der Veröffentlichung unserer Resultate in der Klinik werden wir diese Mitteilungen ausführlicher besprechen.

Eine eingehende pharmakologische Untersuchang des Erythrophleins führten Harnack und Zabrocki (10) aus. Sie konnten das Erythrophlein, seine Salze und Doppelsalze nur orbalten als klare Sirupe, die bei Kalt- und Warmblütern sowohl Digitalin- wie Pikrotoxinwirkung entfalteten. Von Harnack ist später das von Merck hergestellte salzsaure Salz untersucht worden (11). Die Giftigkeit war eine erhebliche: $3 \mathrm{mg}$ töteten eine Katze, $4 \mathrm{mg}$ verursachten bei einem Hunde von $14 \mathrm{~kg}$ schwere Vergiftungserscheinungen. Die Wirkung war die des Digitalins ohne dass, wie bei den älteren Präparaten, klonische Krämpfo (Pikrotoxinkomponente) erzeugt wurden. Die Substanz war feines schwachgelbes amorphes Pulver, das beim Aufbewahren zu einer festen Masse zusammenklebte. Sie wurde chemisch sorgfältig studiert.

Auch von Cushny (21) wurde das Erythrophlein, nebst anderen Substanzen der Digitalisreihe, studiert. Er fand am Sängetierherzen besonders die frequenzhemmende Wirkung stark ausgeprägt.

\section{Technik anserer Versuche.}

Der Ausgangspunkt für unsere Technik war die Versuchsanordnung Jacobj's (12).

Bei dieser werden in das isolierte Froschherz Kanülen eingebunden in die linke obere Hohlrene und in den Bulbus sortae. Der Zuflussdruck von Normal-oder Giftlögung kann variiert worden. Der ausgeworfenen Flüssigkeit steben 2wei Wege offen. Einer fährt zu einem vertikalen Steigrohr, dessen Wassersäule auf dem Quecksilber des Manometers rubt. Der andere Weg führt zam künstlichen Gefässwiderstand, gebildet von einem horizontalen Glasrobr, in welcbem oin genau passender Glasstab mebr oder weniger woit binoingesoboben worden kann. Der Widerstand, den der Kaplllarspalt der austretenden Flässtgkeit gegenüberstellt, ist für eine bestimmte Länge dieses Spaltes konstant. Durch Verlängernng dieses Widerstandes lässt sich der "arterielle" Druck omportreiben, bis der höhere Druck abormals ebensoviel Flüssigkeit durch den grösseren Widerstand presst als der Ventrikel in das System hineinwirit; ein neues Gleiohgewicht ist dann entstanden. Durob Erböbung des ${ }_{n}$ venösen " Zuflussdrucks kana wan das Pulsrolum steigern. Graphisch mossbar sind nun: 1. der arterielle Drack und die Pulsfrequenz, aus der Manometerkurve, 2. das Minutvolum aus der Flüssigkeitsmenge, die ans dem Kapillarspalt strömt. Das Pulsvolüm orbält man aus dem Qootient Minutrolum: Frequenz, die Minutarbeit durch Multiplikation von Minutvolum und arteriellem Druok. 
Eine sinnresche Vorricbtung gestattor des weiteren die graphische Registrierung der Volumänderungen des Ventrikels. Das Herz wird bis zur Atrioventrikularfurcho in einen kleinen Napf mit Nährflüssigkeit getsucht. Der Napf hängt an einem Gummifaden, der sich debut, wenn infolge der Füllung des Ventrikels sicb der Napfinbalt vergrössert. Die Bewegungen des Napfes werden mittelst eines borizontalen Hebols vergrössert auf einem Russzylinder aufgezeichnet. Fängt der Ventriket an, seine Arbeit in einom mebr diastoliscben Stande zu leisten als kurze Zeit zuror, orgibt sich dies sofort aus dem Sinken der Kurve des „Pulsvolumeters".

Das Prinzip dieser Versuchsanordnung wurde von uns festgebalten, in der technischen Ausführung jedoch brachten wir wesentliche Abänderungen an.

Erstens ersetzten wir, wio aus der $\Delta$ bb.1 [Taf.XXIV] ersiclitlıch, die komplizierten Mariotteschen Flaschen für Normal- und Gifllosung durch einfache $9 / 4$ Liter-Flaschen mit seitlichou Tubus und sorgfältig eingeschliffenem, bis etwa $6 \mathrm{~mm}$ vom Boden reichendem Steigrobr. Wir acbteten streng darauf, dass die unteren Enden dieser Steigröbre in genau derselben Horizontalebene lagen, uenn dio beiden Flaschen auf einer wagerechten Lnterlago standen. Bei den ron uns benutzton Gefässeo war dio Niveaudifferenz dieser Enden sicher niobt grösser als $1 / 10 \mathrm{~mm}$.

Die Flaschen stehen auf oinem dreifüssigen Holzscbemel, dessen obero Fläche wittels Stellscbrauben in den Füssen genau wagerecht gestellt werden konnte.

Normallösung und Giftlösung werden aus den Tubi der zwei Mariotteschen Flaschen mittels Gumaiscbläuchen wit Klemmschrauben zu oinem starkev Dreineghahn gofubrt. Die Bohrungen des letzteron waren gleich woit. Mittels eines kurzen Gummiscblauchs mil Klemmscbraute ist das abfübrende Haborobr verbuuden mit der renösen Kanüle.

Der Kanülenbalter, in wolcbem dio zwei lianülen gefasst werden, bestand aus zкoi Ebonitklötzen, welche aufeinander gescbraubt die Kanülen in einer Rinne zwischen sich lassten Das ganze wurde wittels erdes Korken in das Ende eines horizontalen Rohres festgedrückt; dieses Rohr selbst konnte an oinem kleinen sch weren Statir auf und nieder geschraubt werden. Die Einrichtung des Manometers und des Widerstandrohrs war diesclbe wie dio Jacobj'sche. Die ausströmende Flüssigkeit wurde jedoob auf andere Weise geniessen. Jacobj fubrt sie den Wollfaden entlang in ein auf einer Art Wage aquilibriertes Geläss, das sich intermittietond entleert, dadurch emparschnellt und einen Stromkreis schliesst. Wir bängten an den Glasstab einen kloinen T'richter an. Die Tropfen felen vom Trichter auf einen son uns kongtruierten elettrischen Tropfenzabler, welcho wir in Abb. 1 [Taf. XXIV] darstellon und der mittels cines Signales jeden hontakt auf dem Russpapier verzeichnet. Für die $\Delta$ nalyse akut eintretender $\Delta$ enderungen der Herztătigkeit hat die Tropfenregistrierung Vorteile vor der Jacobj'schen Vorrichtung.

Eine woitere $A b$ weichung bildeten bei nnseren Versuchev die Vorrichtungen, welcho dio Höheneinstellung der Nivequs ron Keservoire, Manometer, Widerstandsrobr und Pulsvolumeter elmöglichten. Das einzige, das bei Jacobj's Anordnung stets eine konstanto Höbe beibebielt, war der Napf des Volumeters. Alles ibrige oder gesenkt. Zur Aenderung des venösen Zuflussdrucks woclsselte Jacebj die Höhe der Reservoire, und damit auch des Manometers und des Widerstandrohres dem-Herzen gegenüber. Es iverden also alle schwero Unterteilo der Apparatur dew winzigen Napfon und dem kleinen Herzen gegenüber verscboben. Wir behrten die Sacho nn. Feststebend auch in itrer g̀egenseitigen Lage, waren die Reservoire auf ibrem Holzschemel, das Manometer und das Widerstandsrobr. Das Herz samt den Kanälenbaliern konnten auf allen diesen feststehenden $\Delta$ pparaten bin eingestellt wetden; nacb orfolgter Einstellung wurde die ganze Volumeteranordnung - Napf mit Gummifaden und Hebel 
- omporgesohraubt bıs die Oherfläobe der Flüssigkeıt Im Napf die Atrioventrikulargrenze berührto.

Das Volumeterstativ stand auf einew Holzscbemel. A uf diesem wurde zum Ablesen des Zuflussdruckes ein kurzes Masstäbchen neben dem Herzen vertikal aufgestellt; der oben befindliche Nullpunkt dieses Stäbcbens lag im Kireau der unteren Enden der Steigröhre der Mariotte'schen Flaschen. Auf dieser Skala war la diesel Weise der venöse Drucl direkt abzulesen. Vie ganze gegenseitigo Elnstellung der verscbiedenen Unterteile gelingt mit dieser Apordnung spielend leicht.

Die Einrichtuag unsoros Volumeters ersiobt man aus der $\Delta b b .2$ [Taf. XXIV]. MIt dem naob Jacobj's Angaben gebauton Instrument konnten wir nicht fet tig wirden: die Reibung in den Lagern der Hebelacbse war zu gioss, dor Gurmmifaden batte zuviol störendo Eigenychwıgungen. Die Reibung brachten wir praktisch auf Null zurück, indem wir den Rebel aus Aluminium mit 2 wei scbraubend oinzustellenden vertikalen feinen Stahl. spitzen in bohlon Achatlagern rubon liessen. Wal der Hebol mittels des Gegengerichts gonau horizontal ins Gleiohgewicht gebracbt, so lag der Scb werpunkt des schwingenden Systems in der Unterstützungshinio. Das Gogengewicht wardedann etwas oingeschraubt, der dadarch ganz wenig schwerere lángere $\Delta \mathrm{rm}$ des Hebels jotzt in bolizontaler Lage unterstützt vom Napfe des Volumeters. Dor so gelagorte Hobel folgt den Beregungen des Napfes mit genügender Genauigkeit. Die Ausscbläge des Hebels sind am grössten, wonn dieser um die Horizontallage schwingt. . Eine foine Stellscbraube am Lager des Hobols gestatlet dessen Spitze eben schreibend an die Russfächo anzulegen. Der Hakon-des Naples war mit dem Härchen des Hebels verbunden duich eine kur/e Scblinge aus dünnem Frauenhaar, also nicht durch oinen einzigen Faden. Mit dieser Scblinge beugt man der störendon Torsion $10 m$ Napf und seinem Aluminiumrahmen ror. Den Gummifaden Jacobj's orsetzten wir durch oinen Satz von Ubrfodern vorschıodoner Stärke, aueinandor aufgehängt wio aus der Abbildung orsicbtlioh. Ein derartiges System hat eine konctante Elastizität und stellt sich rasch auf eine Spannungsänderung ein, dio Eigensch wingungon sind also weuig störond, die Inzaul und Stärke der Fodern beberrscht die Grösse der Ausschläge des Ganzen. Der Stauvstab, der das ganzo System trug, konnte, in dem Tubas oines Mikroskops befostigh, witiols dessen grosseñ Triobs auf und nieder geschraubt worden.

Auf der Russfäcbe scbriebon aun 1 das Sigasl der ausgororfenen Troplen, 2 ein Signal für die Markierung von speziellen Eroignissen im Verlaufo des Experiments, wio Einschaltung der Giftlösung, Aenderung des renösen Drucks oder des Glaswiderstandes nsw., 3 zwei Signale für die Żeitmarkierung, eins für die halben Sokunden, das andero für die viertel Minuten, 4 das Manometer mit 5 soiner Abzisse, 6 der Volumeterbebel mit 7 dessen $\Delta$ bzisse. Die Schreiber wurden genau in einer Vertikallinie sobreibend aufgestellt. Das Volumeter schrieb also auf demselben Kymographion wie die übrigea Apparate, in Gegensatz zur Anordnung Jacobj's. Seine Abszisse zeichnete ihro Linio so nahe wie möghich an der Volumeterkurvo selbst, was ein Systolisch-oder Diastolisch berussten Streifon raren 4 Meter lang, die Umlaufzeit war etwa 50 Miauten.

Bei unseren späteren Versuchen haben wir gleichzeitig nit Jer graphischen Registrierung auch das Flektrogramm geschrieben. Aufänglich leiteten wir dieses ab mittels platinierter Platinelektroden aus dem Volumeternapf und dor venösen Kanüle, später benutzten wir unpolarisicrbare Elektroden nach Place. Zugleich machten wir Kinematogramme der Herztätigkeit und $z$ war so, dass jedo Aufnahme von einem Signal sowohl auf dem Elektrogranm wio auf der Russkurvo markiert wurde. Uebcr dio Ergebnisse dieser Versuchsanordnung hoffen wir später ausführlicher zu berichten. 
Die richtige Präparation des isolierten Hęrzens (wir benutzten fast ausschliesslich das Herz des Grasfrosches (Rana temporaria), obschon dieser in Holland ziemlich schwer zu beschaffen ist, aber bekanntlich für die Digitalisgruppe empfindlicher als die Esculenta) ist eine ganz typische Operation. Sie erfordert ziemlich viel Uebung. Vor allem wirkt folgendes nachteilig auf das Resultat: eine zu hohe Temperatur, ungenügende Schonung des Venensinus, unzeitige Verblutung des Tieres mit nachfolgendem Zuklappen der Venenwande, sowie Verstopfen der Spitze der arteriellen Kanüle durch die Spiralklappe des Bulbus Aortae. Am anfanglichen Fehlschlagen der Operation orkannten wir die Unvollständigkeit der Jacobj'schen Beschreibung. Der Wichtigkeit halber möchten wir sie hier etwas mehr detailliert folgen lassen.

Auf einem Froschbrett naob Focke wird der Frosch mit vier Wollfäden fost aufgebunden. Der Mund wird aufgespert und mit einem schmalen scharfen Meissel die Wirtelsäule binten in der Mundhüble mit oinem Schlage durchtrenat; durch das Loch werden Gehirn uad Rückenmark ausgebohrt. Der Blutverlust ist dann minimal, was wichtig ist für die Beurteilung der Herztätigkeit, sobald die Brustböble offen liegt. Den Hautschnitt führen wir von der Symphyse kranialwärts bis etwa zum Sternum; dort wird der Scbnitt V-förmig fortgeführt nacb den Schultern. Auf den umgeklappten V-formigen Lappon kommt später der Ventrikel zu liegen und ist damit für Hautsekret geschutzt. Das Brustbein wird über die ganze Länge gespalten, die Muskeln vom Schultergürtel beiderseitig stıınpf abpräpariert, die Schultergürtel lateral mit der Schere durchtrennt und abgetragen, die unterliegendon Muskeln stampf quer durchtrennt. Jetzt offnet man mit der Schere das Perikard kranialwärts und setzt den Schnitt $z$ wischen den $z w \theta i$ Aorten soweit wio möglich kranialwärts fort. (Binokulare Lupe zu empreblen!)

Zwei nacb den Seiten bis auf die Lungen durch das Perikard gefübrte Schnitte legen das Herz ganz froi. Eine Ligatur wird ut das Fronulum cordis (Plica renae bulbi) gelegt, das Frenulum dorsal von dieser durcbtrennt, das Herz mittels der Ligatur nach oben umgeklappt. Jetzt öffnet man die Banchböble in der Medianlinio, fasst dann auf beiden Soiten die muskulöse Bauchwand und das Baucbfell in der Pinzette und durchtrennt das Peritoneum über der Leber lateralwärts. Wenn man nun suf beiden Seiten die Leber in der Pinzette kaudal wärts luxtert, kann man mit einer feinen Schere die hintere Perikardwand medianwärts bis ganz nabe am lateralen Raade der unteren Hoblveno inzidieren. Unter die Vene, die hier mit dem Perikard verwachsen ist, fährt man eine Hoblsonde bindurch und bringt auf dieser mit der krummen scbarfen Nadel dio Ligatur bindurch, wolche sofort fost geknotet wird. Dio Vene kann nun peripher durchschnitten werden, dic Leber sowie der durcbsobnittene Magen und die übrigen Baucheingeweide werden entfernt. Um die Untorlage des Herzens weniger beweglich zu gestalten, was für das spätere Abpräparieren wichtig ist, verbindet man jetzt die Symphyse mit dem Magenrest durch oine niobt zu stark angezogene Schlinge. Man fasst darauf die Lungen in Schieberpinzetten und durchsobneidet die dorsalen peritonealen Frenula; die Organe können jetzt ganz nabe am Herzen abgebunden und darauf abgetronnt worden.

Nun fübrt man Ligaturen unter beideAorten. Wichtig ist es, dass diese letzteren zuvor von Perikard und Unterlaga ganz frei präpariert werden, wobei Lą̧sionen ron Vorbof und oberen Hohlvenen bei einiger Uebung vermieden werden können.

Die rechte Aborte wird nabe an Trunkus fest unterbunden nnd peripher durchtrennt, um die linke wird eine lose Ligatur gelegt. Das Unterbinden der beiden oberen Hoblvenen wird erleichtert, wenn mau mit einer feinen Schere ganz nabe an kaudalen Rande dieser Venen deren Unterlage schiohtweise durchtrennt. Mit einer foinen Pin- 
zotte mit gekrümmten Spitzen zieht man die Ligaturen mögliobst peripher durcb and tnotet die rechte. Die venöse Kanüle liegt gofüllt fertig, mittels eines kurzen Schlauchs mit Klemmschraube verbunden mit oinem längeren Schlauch, der zu einem kleinen Reserroir mul Näbrflüssigkeit fübrt. Die linke Vene wird, am besten mit einem einzigen Soberenscblag von der richtigen Grösse so peripker wie möglich geöffnet und die Spitze der Kanülo sofort in den Vorhof gefäbrt und dann im riobtigen Stande fest. gebanden. Man ötnet nun die Klemmschraube und durcbspält das Herz unter gerıngem Drack. Die Vene muss dann peripber durobtrenut werden. Jetzt öffoet man mit schrägem Schnitt dio linko Aorta an ibrer Dreiteilang, zerstört die Spiralklappe im Bulbns mit einer feinen Sonde (nicht zu xeit bineinstechen, Vorsicbt mit den Semilunarklappen!) und bindet die Kanüle im riobtıgen Stande mit der Spitze im Bulbus oin, wonn man siob üborzeugt hat, dass die Flüssigkeit obne sichtlicben Widerstand in die Kanüle hineingetrieben wird. Die $\Delta$ orta durchschneidet man peripher und sistiort nun die Darchspülung. Naoh Darcbtrenzung dor Vens oars sup. dextra nabe an der Unterbindung und der Ligatur des Frenulum cordis fasst man boide Kanülen unbereglicb zrischen Vaumen and Zeigeânger der linken Hand und präpariert mit der grössten Vorsicht ohne Zerrung das Herz ron der Unterlage ab; was dabei pon letzterer am Horzen bängeu bloibt, präpariert man bei sparsam gespoistem Herzen vorsicbtig ab, wenn man die Kanülon in richtiger gegenseitiger Lago im Fbonitträger eingehlemm: hat. Es gelingt nicht, die Wand der unterliegenden Lufthöhle ganz abzutragen;-an oiner Stelle ist diese mit dom Vorbof rorwacbsen. Nach Schluss der Klemmscbraube und Lösung der Vorbindung 2 wischen dem karzen und dem längeren Gummiscblauch wird das Horz ins Experimentierzimmer gebracbt ond oinerseits an den Dreiweghabn, anderseits an das gofüllte arterielle Systom angeschlossenr und auf einen vonöson Druok ron otwa $25 \mathrm{nım}$ oingestellt. Man öffot nun den Manomoterbahn and dio Klommsobrauben am arteriellon System, ontfernt den Uebordruck aus dem Steigrobr des Roservoirs mit Normallösung, stellt den venösen Zufluss ber und öffnet schliesslich die Klommschraube zum Widerstandsrobr. Die Füllubg des Vorhofs soll sein wie im Tiere vor der Operation; man regelt sie mittols des venösen Drucks. Der artorielle Druck soll langsam emporgetrieben werden, mit der Länge des Kapillarwiderstandes beherrsoht man hier die Lage. Das Palsrolum soll schliesslich bei einem mittelgrossen Frosob otwa $0,09 \mathrm{ccm}$ betragon. Wean das Herz rubig arboitot, scbraubt man das Volumeter empor, bis die $\Delta$ trioventrikularfurcho dio Oberfäche der Flüssigkeit berührt. Dor Hahn des Manometorsteigrobrs ist ganz offen. . Die Zoit, wolche jetzt verläuft, bis der Druok konstant geworden ist, benutzt man zum Auffangen von 40 Tropfen der Nährlösung, wolche sofort gewogen worden. Wonn der Druct wäbrend mebrerer Minuten gleich bleibt, kann das Kymographion in Gang gesetzt werden.

Man gewinnt bei der späteren Analyse eine Kontrolle der Manometeranweisungen, indem man während der Registrierung einige Malo wit genauer Angabe des Zeitmoments im Protokoll dio systolische Maximal- und die diastoliscbe Minimalböho der Wassersäule im Steigrobr des Manometers abliest. Dazu soll das Nivesu der Atrioventrikularfurcbo auf der Manometerskala bekannt soin; zweckmässig stullt man also vor der Füllung des artoriellen Systems mit der Näbrflüssigkeit das Niveau der Quecksilberoberläcbe ein auf die Höbe des Nivesus der unteren Enden der Steigröbre in den Mariotte'schen Flaschen. Wonn dieses Niveau, das für alle Vetsucho dasselbe bleibt, hekannt ist, kann aus diesem, den renösen Druck und der abgelesenen Höbe der Wassersäule der wirklicho arteriolle Wasserdruck leicht berechnet werden.

Am Ende des Versuohs schreibt man den Nulldruok, nacbdem man bei abgeklemmtem Widerstandsrobr und geschlossenem Habn deq Manometersteigrohrs das Herz ron der arteriellen Kanüle abgetrennt hat.

Zur Kurvenanalyso braucht man schliesslich noch den Inhalt des Manometersteigrobrs pro Zentimeter Höbe zu kennen, welcher sich leicht berechnen lässt. 
Das arterielle System Jacobj's schafft für das Herz vielleicht nicht ganz optimale Bedingungen, da os keine elastische Wand besitzt. Die Differenz zwischen diastolischem Minimal- und systolischem Maximal. druck ist hier viel kleiner als im Tier; die Kammer findet nach der Oeffnung der Semilunarklappen sofort einen höheren Druck vor, braucht jedoch nicht, wie im Tier, diesen Druck während der Entleerungszeit um vieles zu steigern. Der Umstand jedoch, dass das Herz imstande ist unter diesen abweichenden Bedingungen stundenlaug konstant die normale Arbeit zu leisten, macht es für uns ganz sicher, dass dieser Mangel nicht hoch anzuschlagen ist.

Die Analysc der Kurvon gestaltet sich nun folgendermassen: Genau durch jede zweite Viertelninutensignalmarke bringt man eine vertikale Linie und bestimmt hier den Druck aus der Manometerkurve.

Zur Bestimmung des Minutvolums einer bestimmten Minute genügt es nicht die Troplen diesor Minute zu záhlen. Bei einer Druckstoigerung gèht ja ein Teil des Minutenvolums in das Manometersteigrohr, bei einer Drucksenkung gehören nicht alle Tropien zur Flüssigkeit, die das Herz in dieser Minute auswirft. Die für eine bestinmte Minute notwendige Korrektur berechnet man also aus der Druckdifferenz vom Beginn bis zum Ende dieser Minute multipliziert mit dem Gewicht der Flüssigkeit pro $\mathrm{cm}$ Höhe des Steigrohrs.

Dic Tropfen müssen sehr genau gezählt werden. Bei einiger Uebung gelingt es die $Z_{w}$ 'anzigstel zu schätzen.

Die Pulsfrequenz liest man am bequemsten auf der Volumeterkurve $a b$.

Die Minutarbeit erhält man aus dem Produkt von Minutrolum und Mitteldruck der Minute.

Pulsvolun und Pulsarbeit berechnet man, indem man Minutvolum bzw. Minutarbeit durch die Pulszahl dividiert,

Sehr wichtig ist die richtige Zusammensetzung der Nahrflüssigkeit. Die Arabin enthaltende Ringerlösung, deren Bereitung $\mathbf{J} \mathbf{a c o b j}$ und Roemor (13) in ihrer grossen Veronalpublikation beschreiben, hat sich bei unseren Versuchen bewährt. Man verw'ende bei ihrer Bereitung dio reinsten Salze. Wir benutzten die Kahlbaum'schen Präparate „zur Analyse mit Garantieschein". Das Arabid war von Merck. Die Menge Soda, w'elches dieses Präparat zur Neutralisation braucht, ist nicht konstant. Vielleicht ist die Herstellung eine noch nicht genügend gleichmässige. Die fertige Flüssigkeit bewahırten wir gefroren auf. Vor jedem Versuch wurde die benötigte Menge $(600-700 \mathrm{ccm})$ aufgetaut, auf ihre richtige Alkalität kontrolliert und darauf in einem hohen Zylinderglas während -..mier Stunden mit einem kräftigen Sauerstoffstrom arterialisiert. Wir ... nnn n.um in in $n$ Ulh $n$, h und micohton di.xo 
ancunehmen hatte. Diese betrug bei unseren Versuchen etwa 16 bis $18^{\circ} \mathrm{C}$.

Als uns während einiger Wochon das Arabin fehlte, benutzten wir die einfache Locke'scbe Lösung mit $0,1 \mu \mathrm{Ct}$. Traubenzucker. Diese Nährfü̈ssigkeit ist kein gleichwertiger Ersat\%.

An einem Punkte ist das Rezept Jacobj's zur Beroitung der Arabinlösung irreführend. Wählt man die Alkalität derart, dass empfindlicbes Lackmuspapier eben sichtbar geblaut wird, so ist mit einer solchen Losung vichts anzufangen. Lange Zeit haben wir dies zu unserem Schaden erfahren ohne za vorstehen, dass die Lösung und nicht irgend ein Fehler in der Operation an. der schlechton Herzwirkung Schuld hatte. Ein zufalliger Besuch Wybauw's, der im Jahre 1900 Jacobj bei seinen Versucben Hilfe leistete, klärte die Sachlage auf. Wir liaben seitdem den Einfluss kleiner Alkalitätsumterschiede aul die Herztätigkeit näher untersucht. Es stellon sich sehr interessante Tatsachen heraus, über die wir an dieser Stelle oiniges berichten wollen.

\section{Einflass der Alkalitat der Nuhrfllssigkeit.}

Um den Einfluss des Alkalitätsgrades der Durchströmungsflüssigkeit näher zu studieren, machten wir folgenden Versuch: Ungefähr $900 \mathrm{ccm}$ von Jacobj's Ringer-Arabinlösung wurden so alkalisch gemacht, dass 5 cem der Lösung mit 2 Topfen 1 proz. Phenolphthaleiolösung eben sichtbar rosa gefärbt wurden. Diese Flüssigkeit arterialisierten wir mittels Sauerstoffdurchströmung während dreier Stunden. Dann wurden $400 \mathrm{ccm}$ abgemessen und mit 4 Tropfen einer 5 proz. Lisung roń Kristallsoda sorgfältig gemisch. Mit dieser Flüssigketl füllten wir die erste Flasche. Die andere erhielt $100 \mathrm{ccm}$ der ursprünglichen Ringer-Arabinlósung, dèr 16 Tropfen $1 / 10$ Normal-Salzsäurelösung zugesetzt worden waren. Diese Flüssigkeit. reagierto mit Lackmuspapier noch alkalisch, blieb mit Phenolphthalein aber farblos.

Das Herz wurde nach gut beendeter Operation mit der mehr alkalischen Flüssigkeit durchströmt unter einem venösen Druck von $27 \mathrm{~mm}$ und arbeitete konstant, wie auf Kurve 19 (Abb. 3 [Taf. XXIV]) zu sehen ist. Nachden die Herzaktion 11 Minuten lang registriert war, wurde das Herz auf die weniger alkalischo Flüssigkeit umgeschaltet. Sehr bald gestaltete sich die Systole weniger vollständig, die Kammer wurde mehr diastolisch, die Exkursionen kleiner. Auf der graphischen Darstellung ist deutlich zu sehen wie das Minutrolum sinkt. Abor auch die Frequenz bleibe nicht unbeeinflusst. Sie nimmt rasch ab. Infolgedessen siukt der arterielle Druck ganz bedeutend. Nach 21 Minuten erhielt das Herz wieder die mehr alkalische Flüssigkeit und bald folgte oine Wiederherstellung der alten Funktion: die Systolo verbesserte sich, das Pulsvolum wurde grör ar der arterielle Druck stieg an. Auch die Pulszahl verm ' jedoch nicht ganz die alte Höhe erreicht, als $\mathrm{d}$.

keit wicder gewechselt wurde und $d$ :

einsetzte. Die nänl .. I

günstigen EE, l.

Ein 
Wir ersehen daraus, dass ẹne dem Lackmus gegenüber eben alkalische Flüssigkeit nicht ausreicht die Arbeit des Herzens aufrecht zu erhalten. Es ist dazu eine Alkalität notrendig, deren $\mathrm{OH}$-lonenkonzentration (wenigstons für die von uns benutzte Arabin-Ringerlösung) beim Umschlagspunkt des Phenolphthaleins liegt. Wählt man die Alkalität noch grösser, so gerät die Kammer in einen systolischen Krampf, welcher das Pulsvolum stark beeinträchtigt und daınit die Arbeit abnehmen lässt.

Wir möchten hier besonders darauf hinweisen, welch einen grọssen Einfluss ein so klciner Albalitätsuntersehied auf die Wirkung des Herzens ausübt. Wenn man sieht, wie schnell der Ventrikel nit der weniger alkalischen Durchströmıngstlüssigkeit diastolisch wird und nur noch kleine Exkursionen macht und wie rasch der Druck herabsinkt, kann man sich nur darüber wundern, dass ein so eklatanter Effekt einem so kleinen Reaktionsunterschied seine Entstehung verdankt. Die Bedeutung dieses Versuches reicht unserer Meinung nach wcit über den Rahmen dieser Erythrophleinuntersuchungen hinaus. Wir brauchen nur die Versuche Barcroft's (11) über dịe Alkalität des Blutes und die Untersuchungen von Straub, Beckmann, Erdt und Mettenleiter(15) zu nennen um einzusehen, welche Bedeutung es hat, den Eiufluss der Alkalitat auf die Herztätigkeit näher zu erörtern. Fixaktere Experimente zur Erläuterung dieser Frage werden von uns beabsichtigt.

Nach diesen Eryebnissen wàhlten wir die Alkalitat der Lösungen bei unseren Versuchen derart, dass $5 \mathrm{fcm}$ der Jösung von 2 T'ropfen 1 proz. alkoholischer Phenolphthaleinlösung eben sichtbar rosa gefarbt wurden.

\section{Die Wirkung des Giftes ạn das isolierte Froschberz.}

Wir schreiten jetzt zur Beschreibung unserer Erythrophlein Im ganzen rerfïgen wir über $\mathbf{4 9}$ wohlgelungene Expcrimente, eine grosse Zahl, wenn man bedenkt, an wie vielen Klippen die Arbeit bei einer derart komplizierten Anordnung scheitern kann. Von dieser Reihe haben wir etwa 25 vollständig analysiert. Auch diese Analyse raubt sehr viel Zeit, man braucht für sie etwa ebensoviele Stunden als das Her/ Minuten gearbeitet hat.

Wir haben unsere Resultate geordnet $2 u$ einer grossen Tabelle, um uns die Uebersicht iiber die grosse Anzahl von Faktoren, die bei Beobachtung und Analyse berucksichtigt wurden, zu erleichtern. Wir zerlegten zu diesem Zweclie unsere 49 Versuche in 45 Rubriken. Die in letzteren genannten Einzelwirkungen des Giftes werden wir in diesen Ahschnitt systematisch besprechen, möchten jedoch zum besseren Verständnis eine eingehende Beschreibung zweier typischer Experinente vorausschicken. Wir haben dabei absichtlich die Konzentration 1:2,5 Myllionen gewählt, weil bei diesor grossen Verdünnung die Wirkung des Giftes sehr vollständig zeitlich zergliedert wird, dic einzelnen Wirkungen sich also bequem studieren lassen.

Versucb rom 16. 3. 1916, Kurve 16, Rana tomporaria.

Erythrophleinsulfat $1: 2,5$ Millionen.

Das Präparat ist dicht. Das Herz erhält dio Jacobj'scbe Nährnüssigkeit unter einem Zuflussdruck von $20 \mathrm{~mm}$ Wasser. Mit der Normallösung genährt arbeitot.das 
Herz konstant, der arterielle Druck ist $\pm 24 \mathrm{co}$ Wasser. Das Eletrtrogramm ergibt eine Zeit von 0,55 Sek. für die Reizüberleitung von Vorbof auf Kammer. Naob der Umsobaltung auf die Giftlösung wird die Kammer diastolisch, die Systolo weniger vollständig, das Pulsrolum sinkt herab und nimmt, da die Pulszabl nicht ansteigt, Druck, Yulsarbeit, Minutrolum und Minuiarbeit mit berunter [Abh. 4 (Taf. XXIV)]. Die Frequenz fängt scbon früb zu sioken an, was sicb melur oder weniger regelmässig wätrend der ganzen Vergiftung fortsetzt. Uas A.-V.-Interrall ist 1 Min. 30-Sek. nach Begina der Vergiftang 0,58 Sek., nach' 3 Min. 55 Sek. 0,66 Sek.

'Nachdem das Gift 4-5 Min. eingewirkt bat, fängt die Kammer sich zu erbolen an: die Systole wird immerfort vollständiger, während die diastolische Erweiterung ausgiebig bleibt. Dio Folge ist ein Steigen aller Werte. Nur die Frequenz bleibt wiederum unberübrt von der Umwälzung, sie setzt thre Senkung im selben Tempo fort. Das A.-V.-Intervall bleibt in àieser Periode anfänglich wachsend: nach 6 Min. 48 Sek. ist es 0,78 Sek., nach 10 Min. 28 Sek. 0,84 Sek,, naob 16 Min. 9 Sok. 0,86 Sok. Später erbolt die Ueberleitung sich um ein Geringes, wohl infolge der stetig abnolimenden Pulszabl, so dass nach 22 Min. 57 Sek. sie 0,80 Sek. dauert und diese Zoit beibehält, bis sie nach 38 Min. 30 Sek. wieder gestiegen ist auf 0,85 Sek. In dieser Periode der vollständigeren Kammerentleerang ist ein Rascberwerden der Zusammenziebung deutlich sichtbar. Wenn das Stadium orreicht ist, wo die Kamwer sich bei jeder Systole rollständig auspumpt, fängt der arterielle Druck wieder zu sinken ao. Denn die Pulszahl ist in stetiger Abnabme begriffon, welche zwar die Füllungszeit für den Vorbof und damit die disstolische Füllung der Kammer steigert, jedoob nicbt in dem Massso, dass das Minutrolum dadurch boohgehaltón werden kann. Bald bat auch die diastolisohe Erwoiterang ihro Greazo orroiobt: ungesobtet der sinkenden Pulszabl nimmt etwa 34 Min. naoh Anfang der Vergiftung das Pulsvolum nicht mehr zu, bleibt kurze Zeit auf gleicher Höhe, bis von .zwei Umständen sein Absinken eingeleitet wird. Der erste ist die Trägheit, der 2 weite die Unvollständigkeit der diastolischen Erschlaffung. Beide boeinträchtigen die Ventrikelfüllung. Die Ueberleitungszeit nimmt in dieser Phase wieder zu: nach 44 Min. 42 Sok. ist sie 0,89 Sek., nach 49 Mir. 33 Sek. 0,91 Sek. Nach 51 Miu. 45 Sek. kommt oine kurzo Periode rou Systolenausfall, die zu einer kurzdauernden Rhytbmushalbierung führt. Ob diese durah Reizausfall oder sinusurikulare Uoberleitungsstörung oder geringere Reizbarkeit des Vorhofs veranlasst war, entzieht sich unserem Urteil. Das Eloktrogramm liess die Sinasausscbläge niobt erkennen. Nach 55 Min. 45 Sek. kebrt der alte Rbythmus wieder zurüok mit nur noch einzelnen ausfallenden Systolen. Während der Khytbmusbalbierung ist das A.-V.-Interrall verkürzt anf 0,85 Sek., nach ibren Verschwinden stellt der alto Wert sich wieder oin. Wenn 9 Min. später die Frequenz nur wenig gesunken ist, bat die Ueberleitungszeit sioh auf 1,08 Sek. verlängert, ist also seit dem Anfange des Versuchs verdoppelt worden.

Die Rhgthmusbalbierung bewirkt eine geringfügige Vormehrung des Soblagroluns, welobe jedoob bei weitem nicht ausreicht, um das Minutrolum und den Druck im gleichen Niveau zu orhalten. Alle Worte erfabren eine scbarfe Senkung. Dio Wiederberstellung des alten Rhythmus gleicht diese Senkung teilweise wieder aus (ci. Abb. 4 [Taf. XXIV]).

Im Endstadium der Vergiftung, etwa nach der 65. Minuto nach Anfang der Vergiftung, nimmt die Pulszahl stark ab, so dass sie in der 75. Minute nur noch 3-4 pro Minute beträgt. Das A.-V.-Intervall ist jotzt wieder etwas kürzer: 0,86 Sek. Auch das Pulsvolum sinkt in dieser Poriode regelmässig ab infolge des sicb ausbildenden systolischen Kammerkrampfes. In der 66 . Minute wird die elastische Kammerzusamenenziehung. das Kleinerwerden des Kammervolums in der Pause, auf der Volumeterkurro deutlich sichtbar. Diese letztere wird später von uns besprooben werden (S. 378). Der Versuob wird abgebrooben 87 Min, nach Anfang der Vergiftung. 
Versuch rom 5. 1. 1916, Kurve 3, Rana tomporaria.

Erytbrophleinsulfat $1: 2,5$ Millionen.

Bei diesem Vorsuch (Abb. 5 [Taf. XXIV]) arbeiteto das Herz unter einem venösen Druck von $30 \mathrm{~mm}$. Bei der Operation war dia Vena capa sup. sin. beim Einführen der Kanüle eingerissen bis nabe am Sinus renosus, so dass beim Knöpfen der Ligatur ein kleiner Zipfel des Sinus mit eingebunden werden musste. Das Präparat war ganz dicht. Anfänglich arbeitete das Herz kräftig und trieb den arteriellen Druck bis $43 \mathrm{~cm}$ Wasser enpor, dann ersehlaffte aber der Ventrikel allmählich, die Exkursionen wurden rleiner, die Kammer arbeitete im Mittelstand. Beim Ingangsetzen des Kymographions war die arterielle Druckböbe nicht ganz $26 \mathrm{~cm}$ Wasser und sank langsam während der 4 Minuten davernden Normalperiode.

Unmittelbar nacbdem das Gift das Herz erreicht batte, fing das Pulsvolnm an, schnell zu sinken. Der Ventrikel wurde rascb diastoliscb, die Ausschläge kleiner und zeniger kräftig. Die Scblagfrequenz blieb nahezu auf gleicher Höte, arterieller Druck, Pulsarbeit, Minutrolun und Minutarbeit sanken rapid ab. $71 / 2$ Minuten nacb dem $\Delta$ nfange der Vergiftung begann der Umschwung. Das Pulsvolum verbesserte sich wieder, die Kontraktionen wurden kräftiger und bei immer noch gleichbleibender Frequenz. stiegen Druck und andere Funktionen wieder an und erreicbten ein Nireau, das ihre Höhe keim Aufange der Vergiftung bei weitem übertraf. Der Drack stieg bis fast $3 \mathrm{l} \mathrm{cm}$, beim $A n f a n g$ der Vergiftung betrug or $24 \mathrm{~cm}$. Wäbrend der letzten Minuten der Steigerung fand die Diastole deutlich schneller statt, sozasagen explosiv.

In der 19. Minute hatte die Verbesserung der Funktion ibren Höhepunkt erreicht und fing das Pulsvolum wieder zu sinken an. Gleichzeitig stieg die Frequenz ein wenig, so dass auch der Druck noch etwas böber wurde. Als darauf aber die Pulszabl ebenfalls abzunebinen anfing, fiel der Druck ziemlich rasch herab.

In der 25. Minute setzten einige Pulse aus, wobei das ganze Herz stillstand. Infolge der bierdurch beschleunigten Abnabme der Frequenz wuchs das Pulsvolum wieder an, weil wäbiend der längeren Pausen der Ventrikel sicb besser füllte. Es tiat sogar in dieser Hinsicht eine Ueberkompensation em, denn anfängliob sank der Druck weniger rascb berab und erfuhr sogar, als der Rbythmus wiedor regelmässig geworden war und keine weitere Frequenzoerminderung mehr auftrat, bei stark wachsendem Pulspolum ron neuem eine Steigerung. In der 30. Minute.trat infolge des sich ausbildenton Ventrikelkrampfes eino schnelle Senkung des Schlagrolums ein, wodurchrier Druok und die anderen Funktionen rasch heruntergingen. Nacb 49 Min. 20 Sek. erfolgte Stillstand des ganzen Herzens bei rollkommen systolischom Stande der Kammer.

Man muss sich also vorstellen, dass dieses rom Anfang an schlecht arbeitende Herz durch das. Erythrophlein eine kurze Zeit zu kräftigerem Arbeiten veranlasst wird, bald aber wieder anfängt 211 versagen. Dann setzt eine durch das Gift verursachte Senkung der Frequenz ein, wodurch der erschöpfte Ventrikel imstande ist sich zu erholen und das Schlagvolum emporzutreiben. Wenn dann die F'requenzverringerung aufhört, wird auch das Minutrolum und damit der arterielle Druck auf kurze Zeit in die Höhe geführt bis das Systolischwerden der Kammer auch diesem Zuwachs ein Ende macht. Vielleicht kann man in der zweiten Verbesserung der Funktion ein Analogon sehen zu derjenigen Digitaliswirkung in der menschlichen Pathologie, wo das Mittel bloss durch Herabdrücken der Frequenz das Herz zu besserer Funktion befähigt. 


\section{Das Verhalten der Frequenz.}

In diesem $\Lambda$ bschnitt wollen wir nur die Aenderungen des Grundrhythmus besprechen und vorläufig noch absehen von Aenderungen der Pulszahl, die durch Störungen der Reizüberleitung oder der Reizbarkeit verursacht werden.

Eine der auffälligsten Erscheinungen der Erythrophleinvergiftung ist die Abnahme der Frequenz, die wir bei fast allen Experimenten beobachteten. Ein Interschied besteht nur im Zeitpunkt, an dem diese Abnahme eintritt. In weitaus den meisten Fällen erscheint sio schon sehr bald, nachdem das Gift dio Herzhöhle orreicht hat. Einige Fälle gibt es jedoch, wo von vornherein die Frequen\% sich nicht ändert, bis in einem weit fortgeschrittenen Stadium der Vergiftung die Verlangsamung doch eintritt. In einer kleinen Reihe ron Versuchen (7), dio mit stärkeren Konzentrationen (1:100000 bis 1:300000) angestellt wurden, sahen wir eine Beschlounigung des Herzschlags in den ersten Minuten der Vergiftung, welcher jedoch bald die übliche Retardierung folgte.

Die Frage, wo das Gift bei diesen Frequenzänderungen angreift, bedarf einer näheren Erörterung.

Im allgemeinen wird angenommen, dass beim Froschherzen die pulsverlangsamende Wirkung der Digitaliskörper nicht am Vagusapparate angreift, weil Atropin dieser Verlangsamung nicht vorbeugen oder sie aufheben kann. Bekanntlich sind beim Säugetierherzen die Verhältnisse in dieser Hinsicht nicht so einfach. Die Wirkung auf die Frequenz greift hier an am Vagusapparat, durch Atropin wird die Verlangsamung aufgehoben. Der Hauptsache nach greift das Gift am Vaguszentrum an, aber Traube (16) hat hervorgehoben, dass auch nach doppelseitiger Vagusdurchschneidung am vergifteten Herzen eine, wenn auch geringe Pulsverlangsamung übrig bleibt, wolche Traube selber auf das Kouto einer peripheren Vagusbeeinflussung schreibt. Immerhin soll man hier mit der Möglichkeit rechnen, dass in diesem Fall auch eine direkte Wirkung auf den Sinusknoten diese geringe Pulsverlangsamung veranlasst. Jedoch fanden Kothberger und Winterberg (17), dass beim, von seinen Nervenverbindungen völlig gelösten Säugetierherzen ein Teil der Pulsverlangsamung sicher auf Angreifen der Digitaliswirkung am Schrittmacher des Herzens selbst beruht. Sie sahen bei der Vergiftung des nervös-isolierten Herzens anfänglich eine leichte Beschlcunigung des Rhythmus auftreten, hervorgerufen durch eine Erhöhung der reizbildenden Funktion des primären Herzzentrums. Eine solche fördernde Wirkung des Digitalisgiftes wurde von diesen Autoren als erstes Vergiftungsymptom fur alle Zentren des Herzens erkannt. Später in der'Vergiftung wurde der Herzschlag verlangsamt und diese Verlangsamung blieb vom Atropin unberührt.

Greift nun beim isolierten Froschherzen die pulsverlangsamende Wirkung wirklich nur an die reizbildenden Zentren selbst an und nicht an dicjenigen Vagusapparate, deren Wirkung vom Atropin aufgehoben wird?

Von allen Autoren wird behauptet, dass bei Durchströmung des isolierten Froschherzens mit Atropin enthaltender Giftlösung die Puls- 
verlangsamung ebensogut stattfindet wie bei der Vergiftung mit Digitalissubstanzen allein. A uch wir konnten dies in mehreren Versuchen bestätigen, zum Beispiel in einem Versuch rom 11. Febr. 1916 (Kurve 15), wovon wir die graphische Darstellung reproduzieren (Abb. 6 [Taf. XXIV]). Diese Versuche beweisen aber nicht, dass das Digitalisgift gar keinen Einfluss hat

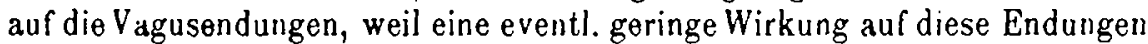
von der viel stärkeren Vergiftung der reizbildenden Zentren verdeckt werden kann. Auch sind die Versuche früherer Autoren nicht beweisend, weil sie mit dem William'schen Apparate gearbeitet haben. Hierbei wird dit zugleich zu- und abführende Kanüle in die Aorta eingebunden und so kommen Sinus und Vorhöre, eben diejenigen Teile, wo die hemmenden Apparate sich befinden, an welchen die Gifte angreifen sollen, nur mangelhaft mit diesen Giften in Kontakt.

Bei dell meisten unserer Atropinversuche wurde das Atropin mit der Erythrophleinlösung dem Herzen zugeführt und man kann bei diesor Anorduung, wie wir oben angeführt haben, keinen schlagenden Effekt auf die Frequenzverminderung erwarten.

In einem Versuche vom 15. November 1915 jedoch vergifteten wir das Her\% mit einer Erythrophleinlösung 1:800000 (Kurve 18, Abb. 7 [Taf. XXV]). Im Anfange der Vergiftung stieg der Druck durch Zunahme des Pulsvolums bei etwas grösser werdender Frequenz. In toxischen Stadium, als alle Funktionen abzusiuken anfingen, wurde das Herz wieder mit Normallösung durchspült. Anfangs blieb die Senkung, auch die der Pulszahl fortdauernd, auch wurde Peristaltik am Ventrikel sichtbar. Als nun nach 33 Minuten keine Aenderungen, insbesondere auch keine Frequenzänderungen mehr auftraten, wurde der Normallösung Atropin in einer Konzentration ron 1:150000 zugesetzt. Dieser zu starken Konzentration ist es woh] zuzuschreiben, dass anfänglich die Frequenz ein wenig abnahm.

Alsbald aber-trat eine deutliche Steigerung auf von 17 auf 23. Die, durch das Erythrophlein veranlasste Verminderung der Pulszahl wurde also durch Atropin teilweise wieder ausgeglichen. Obgleich wir diesem einen Versuche keine zwingende Beweiskraft beilegen wollen, möchten wir doch bemerken, dass er die Vermutung nahelegt, dass beim isolierten Froschherzen die Wirkung auf die Frequenz nicht ausschliesslich gegen die automatischen Zentren selbst, sondern auch gegen den hemmonden Vayusapparat gerichtet ist. Und da wir laut dem Obengesagten Grund haben anzunchmen, dass beim Säugetierherzen diese Wirkung ebenfalls sich richtet auf beide Angriffspunkte, wenn auch im umgekehrten, relativen Verhältnis, so kommen wir zur Vorstellung, dass der vielgenannte Gegensatz von Kaltblüter und Warmblüter in bezug auf den Angriffspunkt der Digitalisbradykardie seine Schärfo eingebüsst hat und nur ein quantitativer Unterschied übrig geblieben ist.

Aus dem oben Angeführten folgern wir, dass die die Pulszahl herabsetzende Wirkung der Digitalissubstanzen, die eine so grosse Rolle spielt in der Therapie, beim Erythrophlein in ebenso starkem Masse gefunden wird. 


\section{Das Vorhalten des Minntvolums.}

Von unseren 49 Versuchen haben wir bei 35 mit genügender Präzisión das Minutvolum berechnet. Bei 16 dieser 35 fanden wir im ersten Stadium der Vergiftung ein Ansteigen des Minutvolums, also auf eine durch das Gift hervorgerufene Verbesserung der Herztätigkeit hindeutet. $\mathrm{Zu}$ diesen Experimenten rechnen wir auch diejenigen, bei delten der Verbesserung eine initiale Senkung, die später von uns besprochen wird, vorausging. Bei 15 Versuchen hatte eine Senkung des Minutvolums als Folge der Vergiftung stattgefunden bzw. eine nicht vollständige Erholung ron der initialen Senkung, bei 4 blieb es im Anfang der Vergiftung auf gleicher'Höhe wie vor der Vergiftung bzw. erholten sich nach der initialen Senkung bis zuı anfänglichen Werte.

Um beurteilen zu können, ob diesen Veräpderungen eine Verbesserung oder eine Verschlechterung der systolischen Kraft des Herzens durch das Gin zugrunde liegen, muss das Verhalten der Frequenz in Betracht gezogen werden. Die drei Faktoren-der Herztätigkeit: Frequenz, Pulsvolum und Minutvolum werden in ihrem gegenseitigen Verbalten von bestimmten Gesetzen beherrscht. Sinkt die Pulszahl, so nimmt das Pulsvolum.zu and kann innerhalb gewisser Grenzen [H of mann (18)] der abnehmenden Frequenz die Wage halten, so dass das Minutvolum nicht zu sinken braucht. Diese Vergrösserung des Pulsvolums ist die Folge einer ausgiebigeren diastolischen Füllung. Bei stark sinkender Frequenz reicht diese Kompensation jedoch nicht aus: die sinkende Pulszahl nimmt das Minutrolum mit herunter (cf. Kurve 1, Abb. 8 [Taf. XXV]).

Bekanntlich bewirkt das Erythrophlein oft cine F'requenzabnahme, deren Folge eine Senkung des Minutrolums sein kann. In vielen Versuchen jedoch tritt neben der Pulsverlangsamung eine durch das Gift bewirkte derartige Verbesserung der Systole auf, dass die Frequenzsenkung kompensiert wird oder noch öfter überkompensiert, so dass trotz der sinkenden Schlagzahl eine Steigerung des Minutvolums auftritt. In diesen Fällen ist die systolische Wirkung des Giftes augenfällig. duer auch wenn bei sinkender Frequenz das Minutvolum abnimmt, kann man oft an der verbesserten und viel rollkommeneren Zusammenziehung des Ventrikels die systolische Giftwirkung spüren, die in diesen Fällen aber nicht ausreicht, bei sinkender Frequenz das Minutvolum hochzuhalten.

$V$ on den 16 Versuchen mit wachsendem Minutrolum trat bei 10 diese Zunahme trotz sinkender Frequenz ein (cf. Kurve 23, Abb. 9 [Taf. XXV]), bei 4 blieb die Frequenz während des Anstiegs des Minutrolums dieselbe wie vor der Vergiftung. Bei diesen 14 Versuchen darf man also von einer die Systole verbessernden Wirkung des Giftes reden. Dies dürfte auch der Fall sein bei den 4 Versuchen, wo trotz sinkender Frequenz. das Minutvolum auf gleicher Höhe blieb. Bei 2 Versuchen jedoch, wo bei steigender Frequenz das Minutvolum anstieg, muss näher untersucht werden, ob eine Verbesserung der systolischen Her/kraft auftrat, und obenfalls wollen wir untersuchen, ob bei den 16 Versuchen mit Verkleinerung des Minutvolums vielleicht doch noch eine Verstärkung der Systole stattgefunden hat. 
Der erste von den $\%$ wei Versuchen, wo Minutvolum und Frequenz boide anstiegen, war einer unserer ersten Fxperimente (19. 6. 1915) und wir können aus dem noch unvollständigen Protokoll keine Besonderheiten über die Kammersystolo und eine event. Verbesserung oder Verschlechterung derselben mitteilen.

Mehr Aufschluss gibt das Experiment rom 15.11.1915 (Kurve 18, Abb. 7 [Taf. XXV]). Wie man aus der Abbildung ersehen kann, steigt nach der Vergifung die Frequenz von 29,3 auf 32. Aber a luch das Pulsvolum steigt! Die Vermehrung des Minutrolums muss also tejlweise von der Verbesserung der vor der Vergiftung unvollkommenen Systole hervorgerufen worden sein. Diese Verbesserung haben wir auch laut des Protokolls gesehen und auf der analysierten Kurve angegeben.

Wie steht es nun mit den $1 \mathbf{j}$ Yersuchen, wo das Minutrolum eine Senkung zeigte?

Von diesen müssen wir drei unberücksichtigt lassen, weil Unregelmässigkeiten des Rhythmus einen Schluss auf das Verhalten des Minutvolums nich zuliessen. In zwei dieser drei Fälle war eine Störung der Roizleitung die Ursache dieser Unrfgelmässigkeiten; dus Block hatte schon vor der Vergiftung bestanden und kehrte durch das Gift gesteigert zurück. Im dritten Falle bestand ein auffäliger, periodisch auftretender Wechsel des Grundrhythmus (Abb. 26 [Taf. XXVII]).

$V$ on den 12 ubrigen Versuchen war die Konzesitration des Giftes bei vier $1: 100000$, bei einem $1: 200000$, bei drei $1: 300000$, bei $z$ wei 1:500000, bei einem 1:1000000, bei einem 1:2500000, d. h. also: bei 10) dieser Fsperimente wurde eine starke Konzentration, bei nur 2 eine schwache angewandt.

Bei diesen starken Konzentrationen tritt die tonische Kammerkontraktur durch das Gift sehr bald in den Vordergrund, so dass nach kurzer 'Leit schon eine deutliche Erschwerung der Diastole auftritt, wodurch das Minutvolum rasch herabsinkt. Beim optimal arbeitenden Herzen, wo die an sich schon nahezu vollständigo Zusammenziehung nicht rom Gifte gebessert werden kann, ist der Einfluss auf die Ventrikelexkursion eine ausschliesslich toxische: nach oiniger Zeit verkleinert sich die diastolische Erschlaffung (Kurve 4, Abb. 10 [Taf. XXV]).

Auch wenn bei den stärkeren Konzentrationen die später zu besprechende initiale Senkung des Minutvoluns stattfindet, welche wir bei den drei Versuclien wit der Verdünnung 1:300000 und bei einem mit 1: $\mathbf{5 0 0 0 0 0 ~ b e o b a c h t e n ~ k o n u t e n , ~ k a n n ~ n a c h ~ d e m ~ A u t h o ̈ r e n ~ d i e s e s ~ i n i t i a l e n ~}$ Stadiums die Wiederherstellung der Herzaktion nicht vollkommen sein. Wohl landen eine Verbesserung der Systole und Ansteigen von Druck und Minutrolum nach der initialen Senkung bei diesen Versuchen stat, aber bald trat infolge der hohen Konzentration des Giftes die Erschwerung der Diastole auf, bevor die Erholung sich. voll ausgebildet hatte, so dass Druck und Minutvolum sich wieder senkten. Ein Beispiel hiervon bilden die Kurven 7 (Abb. 11 [Taf. XXVI]) und 17 (Abb. 12 [Taf. XXV1).

Von den zwei Fällen mit schwacher Giftkonzentration war bei einem (1: 1000000, Kurve 11, Abb. 13 [Taf. XXVI]) die Herzkammer schon vor der Vergiftung ziemlich systolisch, so dass oine systolische Verbosserung 
durch das Gift unmöglich war: das Minutrolum sinkt der Frequenz parallel. Beim anderen Versuch $(1: 2,5$ Mill., Kurve 16, Abb. 4 [Taf. XXIV]) tritt eine starke initiale Senkung auf, aus welcher trotz sinkender Frequenz eine fast vollkommene Wiederherstellung der alten Funktion stattendet. Die Frequenzsenkung war hier wohl zu stark, um eine weitergehende Verbesserung von Druck und Minutvolum zu gestatten.

Nach dem näheren Studium des Verhaltens des Minutvolums kommen wir zu dem Schluss, dass wir immer, wenn nicht durch zu hohe Konzentration des Giftes das toxische Stadium zu früh auftrat oder das Herz schon vor der Vergiftung optimal arbeitete, eine durch das Gift bewirkte Verbesserung der systolischen Herzkraft haben wahrnehmen können.

\section{Das "Initialstadium".}

Wenn man, wie wir dies taten, mittels einer gefärbten Flüssigkeit festgestellt hat, eine wio grosse Tropfenzahl aus dem Widerstandsrohr fliessen muss, bevor nach Umschaltung des Drejweghahns dio gefärbte Lösung den Vorhof erreicht, so kann man bei jedem folgenden Versuch mittels dieser Tropienzahl den Zeitpunkt, an dem die erythrophleinhaltende Mischung das Herz erreicht, mit genügender Genauigkeit ablesen. Auf diese Weise liess sich feststellen, dass in vielen unserer Versuche dieser Zeitpunkt durch das Einsetzen einer auffälligen Reihe von Erscheinungen markiert war. Man sieht die systolische Zusammenziehung des Ventrikels weniger vollständig werden, so dass die Kamner einen ausgesprochen diastolischen Stand annimmt und das Schlagvolum ständig herabsinkt. Bald sinkt auch dio Pulszahl, bald hält sie sich in gleicher Höhe. Vereinzelt beschränkt sich die Wirkung auf die Frequenz, so dass dann sogar ibre scharfe Senkung eine temporäre Vergrösserung des Pulsvolums herbeilühren kann. Häufiger ist das Umgekehrte der Fall: Finschräukung des Pulsvolums bei gleichbleibender Frequenz. Letztere Kombination nebst einem diastolischen Stande des Ventrikels möchten wir als Typus unseres "Initialstadiums" aufstellen. Am deutlichsten war sie ausgepragt bei der kleinsten von uns benutzten Giftkonzentration $1: 2,5$ Mill. Hier ist, falls ein Initialstadium vorhanden ist, regelmässig das Pulsvolum beeinträchtigt, die Frequenz wird nicht oder kaum berührt. Ein Beispiel geben wir in der Kurve 24, Abb. 14 [Taf. XXVI]. Zum negativ chronotropen Effekt scheint das Herz grössere Konzentrationen zu brauchen: deutliche initiale Bradykardie sahen wir vor allem bei einer Giftstärke von 1:300000 auftreten.

Es ist einleuchtend, dass ein Sinken von Frequenz und Pulsyolum oder auch von letzterem allein auch die übrigen Funktionen; Minutrolum, Druck und Minutarbeit herabdrückt. Ein Blick auf die Kurven 16 und 21, Abb. 4 [Taf. XXIV] und 15 [Taf. XXVI], gibt davon ein klares Bild.

Die Dauer des beschriebenen Initialstadiuns ist keine grosse. Die Erholung erfolgt innerhalb - weniger $(2-7)$ Minuten. Dabei wird die Systole rasch vollständiger, wahrend die Erweiterung in der Diastole sehr ausgiebig bleibt. Allo Werte steigen wieder an. Eine Ausnahme macht dabei oft die Pulszahl, deren Erholung oft ausbleibt oder in un- 
vollständiger Weise erfolgt. Die erste Wirkung des Giftes ist ja, wenn man absieht von den initialen ${ }_{n}$ Kontakterscheinungen ${ }^{\alpha}$, gegen den Schrittmacher des Herzens gerichtet und in wenigen Minuten, die das Initialstadiam zum Abklingen braucht, lann diese resorptive Wirkung schon eingeleitet sein. Diese fortschreitende Verringerung der Pulszahl kann bewirken, dass das Minatrolum und der Druck nicht mehr aus dem Tal herauskommen, dass also trot\% des wachsenden Schlagrolums dic Leistung pro Zeiteinheit sich nicht oder ungenügend erholt. Man sieht dies bosonders bei den höheren Giftkonzentrationen, z. B. $1: 300000$, während bei den noch höheren $(1: 1000000)$ die Erholung ausbleibt, weil durch die starke systolische Wirtung das Initialstadium sofort in das toxische übergeht, die diastolische Erweiterung also schon früh eingeschränkt wird.

Wenn wir auch ganz leichte Aenderungen von Frequenz und Pulsvolum mitrechnen, so haben wir etwa in der Hälfte unserer Versucbe die beschriebenen ninitialen ${ }^{*}$ Erschoinungen boobachtet. Ein voll ausgeprägtes "Initialstadium" war jedoch ungleich seltener.

Die Antwort auf die Frage nach dem Wesen dieser eigentümliehen Erscheinungen ist nicht leicht zu geben. Sie sind denen der Muskarinvergiftung sehr ähnlich. Denn auch dort findet man ein Diastolischwerden der Kammer mit stark sinkendem Pulsvolum und mehr oder weniger abnehmender F'requenz, auch dort eine Erholung, wenn die vergiftende Bonzentration nicht zu gross ist. Man würdo also geneigt sein, sie, wie bei jener, einer Vagusreizung zuzuschreiben. Uns ist die Uobereinstimmung mit dem Muskarin zu spät aufgefallen, sonst hätten wir sicher einige Male versucht, dem Initialstadium durch vorhergehendo Atropiuisierung vorzubeugen. Einen Umstand jedoch gibt es, der es uns von vornherein etwas unwahrscheinlich macht, ob die Erblärung in dieser Richtung zu suchen ist. Daśs ist das ungemein rasche Einsetzen des ganzen Komplexes. Unsere Versuchsanordnung erlaubte nicht, den Zeitpunkt, an dem das erste Giit das Herz erroicht, nit mathematischer Genauigkeit festzustellen. Auch lässt die Art der Analyse, die selbstverständlich nicht von Sekunde zu Sekunde ausgeführt werden konnte, den Anfang des Stadiums auf unseren graphischen Darstellangen ungenügend genau hervortroten. Mán darf jedoch ruhig den Zeitaufwand, den das Herz braucht, um auf die ersten Giltsparen sichtbar zu reagieren, auf nicht mehr als eigige Sekunden stellen, und es fragt sich jetzt, ob diese Zeit genügt, um eine Resorption des Giftes zur Erklärung der Erschcinungen heranzuziehen. Möglicherweise haben wir es mit einer reinen Kontaktwirkung zu tun, Kontakt mit den sensiblen Nervenendigungen zwischen den Endothelzellen des Endokards. Auf die Bedeutung dieser ron Smirnow Hoijmans, und Demoor und Schmidt (19) beschriobeneu Elemente hat $\mathrm{W}_{\mathrm{y}} \mathrm{bau}$ (20) hingewiesen. Er beansprucht für sie die wichtigo Rolle für die Pharmakologie des Herzens, welcho sio wahrscheinlich während des Lebens zu spielen haben. Man könnte sich nun vorstellen, dass diese Ròlle in einer reflektorischen Regulation vom Tonus der Kammerwand bestohe. Ihre Roizung würde einer Erschlaffung des Muskels entsprechen. Eine solche Einrichtang wäre zweckmässig: eino grössere Vorhoffüllung bedingte dann mittels Reizung dieser Endigungen 
automatisch eine Tonusverminderung des Ventrikels, damit dieser den grōssoren Inhalt ohne Kraft vergeudung des Vorhofs aufzanehmen befähigt wärde. Die Ganglienzellen und die efferenten Bahnen dieser Reflexpeuronen könnten Vaguselemente sein. In dieser Weise erhalten die Initialerscheinangen der Erytbrophleinwirkung eine ungezwungene Erklärung: sle wären demnach der Beeinflussung des Vagussystems zuzuschreiben, aber doch in besonderer Weise, indem sie nicht von einer dirokten, sondern ron einer reflektorischen Vagusreizung ausgelös̄t wärden. Diese Erklärung hat auch insofern etwas Bestecbendes, als die heftig reizende Wirkung des Erythrophleins auf sersible Nervenendungen längst bekannt ist.

Sie war ja die Ursache, dass die Anwendung der Substanz als Lokalanästhetikum aufgegeben werden musste.

$\dot{N}_{\text {ach }}$ einigen Minuten treten die resorptiven Wirkungen des Giftes in die Erscheinung: die vollständigere und raschere Systole der Kammer, die das schlaffe Herz aus dem Initialstadium emporbringt. Auf letzteres weist, nur noch der geringere Tonus des Ventrikels in der Pause hin. Die daron abhängige grössere diastolische Füllung verursacht unter Mitarboit der Systolenverbesserung die Vergrösserung des Schlagvolums im therapeutischen Stadium.

Vom entwickelten Gesichtspunkte aus die Rọlie des V'agussystems beim Entstehen des Initialstadiums näber $2 u$ studieren, erscheint eine dankbare Aufgabe. Vor allem scheint uns eine genaue bistologische Untersuchung, nebst Kersuchen am atropinisiorten Herzen bierzu notwendig. Vielleicht mag auch das Studium des Einflusses einer kurzdavernden Kokainisierung des Endokards sich dazu eignen.

Eine weitere Frage ist, ob im Herporrufen der initialen Erscheinúngen das Erythrophlein vereinzelt dastebt oder ob auch den anderen Gliedern der Digitalisgruppe diese Eigenschaft zukommt. Der Umstand, dass man in der Literatar unser Initialstadium nicht nennt, braucht dabei nicht ausscblaggebend zu sein, denn die Unsitte, für das Studium der Digitaliswirkung am Froschherzen Sinus und Vorhof mit allen wich́tigen darin gelegenen Elementen mehr oder weniger auszuschalten, indem man sich des Verfahrens von Williams oder Kronecker bedient, ist allgemein verbreitet und es ist sehr wohl möglich, dass am Zustandekommen des Initialstadiums die Wirkung auf den Ventrikel selbst gar nicht beteiligt ist. Auf die übliche Ausschaltung ron Sinus und Vorhof kommen wir boi der Besprechung des diastolischen Stillstandes noch zurück.

\section{Die Elastizitatsunderang der Kammer.}

Mit den anderen Digitaliskörpern teilt das Erythrophlein die Eigenschaft in den späteren Stadien der Vergiftung die Kammer in einen systolischen Kramplzustand zu versetzen. Dieser beeinträchtigt die passive Kammerfüllung und erschwert die Aufgabe des Vorhofs, sich vollständiz zu entleeren, damit das Schlagvolum emporgehalten werden kann. Ein Beispiel gibt die Volumeterkurve auf Abb. 16 [T'af. XXVI].

Hier sieht man die Linie, welche während der Pause der Herzaktion rom Volumeterschreiber geschrieben wird, in ein immerfort höheres 
Niveau rücken, sich also der Abszisse nähern. Es bedeutet dieses eine unvollständige Diastole der Kammer. Die Gipfel der Systolen bleiben jedoch ungefähr im selben Nivean, d. h. das Ventrikelvolum ist am Endo der Systole stets dasselbe, also minimal. Die Höho der Exkursionen nimmt demnach ab. Auf dieser Kurve, aber besser noch auf der rom 10.1.1916 (Abb. 17 [Taf. XXV]]) lässt sich auch beobachten, wenn man die 0., 20., 53., 57., 82. und 83. Minute vergloicht, wie die Erhebung, welche der Kammersystole entspricht, immer mehr zurücktritt gegenüber der Senkung, welche die Folge von der Kammeransdehnung durch die Vorhofssystole ist. Das heisst also, dabs die Kammer sich in der Diastole immer schwerer ausdehnt und erst die Vorhofskontraktion ihr Volum akut wachsen lässt. Folgt dann die Systole der Kammor, so wird ihr Inhalt vollständig ausgetrieben, aber die Erschlaffung folgt darauf kaum oder nicht, bis die neve Kontraktion des Vorhofs einsetzt. Schliesslich wird auch die von letzterer abhängige Senkung kleiner: der Vorhof erlahmt. Das Ende ist, wic ersichtlich, der systolische Stillstand der Kanmer mit gelàhmtom, maximal dilatiertem Vorhof (Abb. 18 [Tal. XXVHI]).

Selbstverständlich sinken alle Werte im Stadium des systolischen Kammerkrampfes rasch herab. Beispiele findet man in den Kurven 6, 7, 13 und 17 (Abb. 19 [Taf. XXVII], 11 ['Tal. XXVI], 5 [Taf. XXIV] und 12 [Taf. XXVI]).

Wir möchten hier die Aufmerksamkeit auf eine Erscheinung lenken, deren Beobachtung wir dem richtigen Arbeiten unseres Volumeters verdanken. Es ist dies, was wir das "Elastizitätsphänomen" genannt haven und das wir bisweilen im Stadium des systolischen Kammerkrampfes auftreten sahen. Man findet sie in der Abb.. 20 [Taf. XXVII] graphisch dargestellt.

Augenfällig ist auf dieser das Steigen der Volumeterlinie $z$ wischen je zwei Systolen. Das bedeatet also, dass in der Pause der Herzrevolution das Ventrikelvolum kleiner wird, die Kammer treibt ihren Inhalt heraus und wird von der neuen Vorholskontraktion völlig leer porgefundent. Der Vorhof erweitert dann die Kammer akut, diese folgt richtig nach mit ihrer Systole, aber erweitert sich jetzt - und das ist das Merkgürdige an der Sache -, wie wenn es gar keinen systolischen Krampizustand gäbe: man sieht die Volumeterlinie sinken bis zum Niveau, das am Höhepunkt der Vorhofsystole eingenommen wurde. Erst im Laufe der Pause steigt die Kurve und kehrt der Kammerbrampf \%urück. Mit anderen Worten: der von der Vorhofsystole überwundene Ventrikelkrampf ist nicht gleich nach Ablauf der Kammerkontraktion wieder da. Es sind demnach der Ventrikelkrampf und die eigentlicke Kontraktion zwei verschiedene Vorgänge, die nicht auf derselben Gruodlage fussen. Will man sich die Sache chemisch vorstellen, so müsste man sagen, dass die chemische Reaktion im Muskel, welche die eigentünliche Elastizitätsänderung zur Folge hat, von amderer Art sein muss als die, welche von der eigentlichen Kontration die unmittelbare Ursache ist, da jene, im Gogensatz zu dieser letzteren, sich nicht hervorrufen lässt durch den voın Vorhof kommenden Reiz. 


\section{Das Verhalton dor Reizliberleitnng and der Roizbarkeit.}

Wir hatten bei unseren Versuchen oft dio Gelegenheit, die negativ dromotrope Wirkang des Erythrophteins iu studieren. In etwas veniger als der Hälfte anserer Versuche finden wir das Auftreten von Systolenausfall in unseren Protokollen erwähnt. Wir wollen hier ganz ausser Besprechang lassen, ob diesen Ausfällen eine Störung der reizleitenden Funktion des Herzens zugrunde lag oder ob wir dio Ursaehe der Arrhythmie suchen müssęn in einer Abnahmo der Reizbarkeit des Hercmuskols: wir haben die Beeinflussung der Reizbarkeit durch das Gift nicht näher untersucht. Wenn wir auch von Block redén, beabsichtigen wir damit nicht, in dieser Frage irgend ein Urteil abzugeben.

In vielen unserer Versuche mit regelmässig zurück kehrendem Systolenausfall stand das ganze Herz still. Die Pulsationen des Venensinus konnten wir nicht registrieren, in einigen Fällen jedoch war die Sinuswirkung deutlich sichtbar and kormten wir beim Stillstand ron Vorhof und Kammer das regelmässige Weiterpulsieren des Sinus beobachten. Auch Ventrikelsystolonausfall, wobei Sinus und Vorhöfe regelmässig woiter arbeiteten, lam oftmals vor und in einigen Fällen konnten wir ein kombiniertes'Bild beobachten: der Sinus schlug regelmässig weiter, jeder dritte Impuls warde aber nicht zu don Vorhöfen hinübergeleitet. Von den Vorhofkontraktionen war wiederum nur die Hälte von einer Ventrikelkontraktion gefolgt. Es bestand also oin 3-2 sinvaurikuläres ond oin 2-1 atrioventrikuläres Block. In den messten Fälen aber bestand entweder sinusurikuläres oder atrioventrikuläres Block. Letzteres überwiegt bei unseren Blockfällen: bei etwas mehr als der Hälfte trat die Leistungsstörung $z$ wischen Vorbof lind Kammer auf.

Einige Male war schon por der Vergiftung eine Leitungsstörung vorhanden, die immer nach kürzerer oder längerer Durchströmung mit Normallösung wieder verschwand. Wurde nun aber die Giftlösung eingeschaltet, dann traten die Blockerscheinungen immer wieder auf, in einigen Fällen fast unmittelbar, nachdem das Gift das Herz erreicht hatte (Kurve 3, Abb. 21 [Taf. XXVII]). In diesen Fällen, wo trotz der stets äusserst schonend susgefährten Operation doch eine Schädigung der reizleitenden Elemente stattgefunden hatte, können wir also deutlich eine gewisse Affinität des Giftes für das beschädigte Leitungssystem erkennen, welche für alle Digitalisgifte längst bekannt ist.

Ein Beispiel von einer ,initialen" Störung der Reizüberleitung findet man in der Karve 10, Abb. 22 [Taf. XXVII].

Einmal haben vir bei vollständiger Blockierung der Reizleitung zwischen Atrium und Ventrikel eine richtige Dissoziation gesehen, wo also der Ventrikel in seinem eigenen Rhythmus weiter schlug. In Abb. 23 [Taf. XXVII] geben wir die Reproduktion einiger Elektrogramme. Das bekannte Bild der Interferenz ron Vorhof- und Kammerrhythmus, wie es bei der atrioventrikulären Dissoziation auftritt, ist deutlich siohtbar. Das Herz war hier vergiftet mit einer Verdünnung ron $1: 500000$ und war, was vielleicht von Interesse ist, ron einer Rana esculenta. Merkwürdigerweise trat das Bild der Dissoziation hier ganz plötzlich beim im normalen 
Rhythmus schlagenden Herzen anf (Volumeterkurve, Abb. 24 [Taf. XXVIT]), ohne dass zavor eine Störung in der Reizleitung stattgofunden batte. Später trat, wie aus der Abbildung ersichtlich ist, 2-1 Block auf. Jedenfalls müssen wir in diesem Falle eine Erböhung der reizbildenden Wirkang ventrikulärer Herzzentren onter Einfluss des Giftes sehen.

Was die so oft beschriebenen Luciani'schen Reithen anbelangt, die auch ron uns im letzten Stadium der Vergiftung beobachtet sind, so wollon wir. uns darüber kurz fassen. Nur vollen wir erwähnen, dass oft die Vorhöfe pährend des Ventrikelstillstandes weiter schlugen, wie auch de Boer ${ }^{\prime 22}$ es kürzlich am Froschherzen in situ wieder beobachten konnte und wie an den in Abb. 25 [Tal. XXVII] abgebildeten Volumeterkurrenabschnitten deutlicb zu sehen ist. In einem anderen Falle schlugen anfänglich nach einer Luciani'schen Reihe die Vorhöfe weiter, um bald awoh still zu stehen. Bei jeder Reihe wiederholte sich dasselbe Spiel. Wenn also das Auftreten der Reihen auf einer Erregbarkeitsverringerung der Kammermuskel beruht, müssen wir durch diesen Versuch auch auf eine analoge Wirkung des Giftes auf die Vorhofsmuskeln schliossen, wenn wenigstens die Vorhöfe nicht aus lauter mechanischen Gründen bald nach dem Ventrikelstillstand ebenfalls zum Stillstand kommen.

An dieser Stelle wollon wir noch einen Versuch erwăhnen, wobei das Hers mit einer Lösung des Giftes ron $1: 2,5$ Mill. vergiftet wurde und wobei einige Minuten pachdem das Gift das Herz erreicht hatte, dieses plötzlich mit viel grösserer Frequenz zu scblagen anting. Bald darauf war aber das alte Tempo wieder zurückgekehrt. Dieser Wechsel des Rhythmus trat et sas spater fortwährend in die Erscheinung, so dass nach einigen langsamen Schlägen unvermittelt ein schnelles Tempo auftrat, wobei Vorhof und Kammer immer in der normalen Weise zusammenarbeiteten und das nach kurzer Zeit wieder einem langsameren Platz machte. So wechselte der Rhythmus von etwa 5 oder noch weniger Schlägen pro Minute auf 30 oder mehr. Dies dauerte fort bis zur 85. Minute.

Einen solchen Wechsel reproduzieren wir in der in Abb. 26 [Taf. XXVU] abgebildeten Volumeterkurve. Es ist deutlich $\mathrm{za}$ sehen, wio der Ventrikel beim schnellen Rhythmus allmählich einen mobr systolischen Stand einninımt. Später trat Gruppenbildung auf, wobei in den l'ausen deutlich Wühlen und Wogen der Kammermuskulatur, auch in der Volameterkurre zu schen war.

\section{Der diastelische Stillstand.}

Der systolische Stillstand ist das typische Ende der Vergiftung mit Digitalissubstanzen. Dio Neigung zum systolischen Stande ist der Ausdrack der Vergiftung der Herzmuskel selbst. Ist sie woit genug ansgobildet, so sermag der Vorhof bei seiner Zusammenziehung die Kammer nicht mehr zu dilatieren, er wird sozusagen überfültt and ertahmt schliesslich. Damit hat die ganze Herzarbeit ein Enda Dass damit jedoch das Herz nicht tot ist, wissen wir aus dem bekannten Versuch Schmiedeberg's. 
Mit der Erythrophleinwirkung am isolierten Froschherzen ist dem nicht anders. Auch hier finden wir, selbst bei den geringsten Konzentrationen, wenn diese nur genügend lange einwirken, das Systolischworden der-Kammer. Stelrt am Ende letztere still, so ist es im systolischen Stande.

Gibt es nebon diesem "regelmåssigen" systolischen Stillstande nun auch noch einen diastolischen? Bevor wir diese Frage für das Erythrophlein beantworten, möchten wir die Fragestellung weiterfassen und zwar: unter welchen Umständen findet man bei der Vergiftung mit Digjtalissubstanzen diastolischen Stillstand und worin ist das Wesen dieses Stillstandes begründet?

Dio Entdeckung dos diastolisoben Stillstapdes stammt bekanntlich ron $J_{\text {acobj }}$ (23). Er rergiftete das isoliorto, am William'sohon Apparat ornäbrto Froschborz mit Holloborein, abor niobt in der übliobon Woiso, indem or das Gift der DurcbströmungsQüssigkeit zusotzte, sondorn ron der Aussenozobe bor, wozn or das Präparat in dio Giflösung ointauchte. Er sah nach oiver Poriode ron stärter ausgeprägten Diastolon das Herz in aasgosprochenster Diastole stillstehen. Im Vorlauf ron otwa 1 Stundo wurde obve rhythmischo Bowogong allmäblich dio typische Digitalissystolo oingenommen.

Wyban (24) hat daranf dio Erscheinang naher untersucht. Er sah dio Pulszabl sinten, dio Disstolen wardon sebr lang, dio Systolon stark. Das Schlagoolum orrejcbto des Maximum, wäbrond dio Arboit durob dio rorminderte Pulszahl fast auf Nall sant. Bald biolt dio Diastolo an und dor Ventrikel stand still. Der Vorbof setzte soine rhytbmischen Bewogungen jodoch fort. Nach oiniger Zoit warde der stillstehende Ventrikel systolisch. Wurde der Versuch an einem atropinisiorten Herzen angestollt, so nabm obenfalls dio Pulszabl ab, das Schlagvolum za, der diastolischo Stillstand blieb jedoch ans and nach langer Zeit folgte das Systolischwerden und der systolische Stillstand. Wy bau folgort bieraus, dass der diastolische Stillstand bei der änsseren Applikation von oiner Hemmang (durch Koizang des Vagusapparates) abhängig ist. Diese Beobachtungen ron Jacobj and W y ba a w haben viel Staub aufgewirbelt. Eine befriodigendo Klartoit babon die bezäglichen Veröffontlichungon jodoch vicht gobracht.

Huldschinsty (25) versuobte die Hemmungstheorie von Wy bau zu stützen, indem er in die Porikardböblo des Froschberzens Helloborein in oiner Menge injizierte, die an und für sicb nictt gonügte, disstolisobon Stillstand zu bowirken. Roizte or nun die Vagi mit einer Roizstäke, die obenfalls edloin nioht zum selben Z reichte, so gab die Summiernag der beidon Faktoren don diastolischen Stillstand. Dass dio Addition stattindet, ist nach Huldochinsky der Berois dafitr, dass boido Einfliłsse derselben $\Delta r t$ sind, dor diastolische Stillstand also in Reizung des Vagas. apparates begründet ist.

Ganz verworfen wurde dio Hemmungsthoorie von Benedicenti (26). Vergiftete er nämlich das am William'sohen Apparat arboitende Frosobberz ron aussen bër mit Stropbanthin + Atropin, so konnte das $\Delta$ tropin nicht, wio W yba a bebauptete, dem diastolischen Stillstande rorbeugen. Dieser kam ebenao gat zustande wio obre Atropin. Der Autor wollte nun den Untersobied dor Wirkang bei innerlicher und bei ausserlicber Applitation orkläron aus Verschiedenbeiten in der $\Delta$ nordnung der innoren und der äusseren Muskelfasorn des Herzens. Dio Wirkung des Giftes an der inneren Schicht sollte systoliscben, die an der zusseren diastolischen Stillstand rerursacben. Diese Theorie wurde sogar ron Sobmiodeberg selbst in sein Lebrbuch übergenommen. Eine starke Erschütterung orfuhr sio durcb dio Arbeit Werschinin's (27), der darauf hinwres, dass auch boi der Fergiftung des Horzens in situ mit sebr kloinen Giftmengon 
dieses biswoilen disstolisch stillstehen konnte. Von einer äusserliobed Applikation ist bier natürlich gar keine Rede. Sogar boi der innerlichoo Applitation am isoliorton Temporarienberzen war der Stillstand diastoliscb, weon nar das Gift in dor Ringorsoben Lösung gonügend verdünnt wurde. Atropia hatie darquf koinen Eịnfluss. Ersetzte or einen Teil der Ringer'schen Lösung durob Kaninohensernm, so neigte die Kammer mehr̈ zum systolischen Stillstand, d. h. dieselben Giftkonzentrationen, die in der Ringer'schen Losung diastolisoben Stillstand erzengt batton, riofen jetzt systolisoben bervor. Werscbinin vermutet, dass die Gegonwart rou Sorumbestandteilen das Findringen des Giftes in die Horzwand fördert, so dass die Elemente, dio den systolischen Stillstand bewirken, rasther erreicht werden. Bei geringeror Konzentration bzw. langsamerom Eindringen des Giftes bönnte denn der engriffspunkt für den diastoliscben Stillstand fräber erreicbt werden. Diese Erklärungen waren Feniger befriedigend als die Deatung, die Holsto (27) von Wersobinin's Beobacbtungen gab. Letzterer verşuchte die neven Tatsacben in den Rabmen der Zweiscbicbtentheorio von Benedicenti und Schmiedeberg zu drängen, indem er ron der Beobachtung ausging, dass das am William'schen $\Delta$ pparat ron Ringer'scher l,ósnng eruāhrte Herz bald anfängt, die Flüssigkeit durch seine Wand siokern zu lassen, im Gegensatz zum Herzen, das mit einer Flüssigkeit gespeist wird, die Blut oder Gummi enthält. Das Durcbsickern der Flüssigkeit und der diastolische Stillstand werden also rom selben Umstand bogünstigt, nämlich vom Feblen kolloider Sabstanzen in der Näbrlösung. Bei dieser abnormen Durchlässigkeit wird die äussere Moskolschicbt rasch vom Gift orreicht und danit ist dio Bodingung für den diastolisobon Stillstand gegeben. Bei grosserer Giftkonzentration erfolgt der systolische Stillstand, woil die innere Muskelschicht schon vergiftet ist, beror das Gift Zeit hat, dio diasto. lische Scticht zu orreichen. Eothiolt die Flässigkeit Blut oder Gummi, so war der Stillstand ausnabmslos ein systolischer. Hierzu soll jedoch bemerkt werden, dass die Giftkonzentration immerhin noch so gross war, dess der Stillstand spätestens nach I Stunde eintrat. Eine geringere Konzentration bätte vielleicht im Sinne Worgcbinin's in aoch längerer Zeit zum diastoliscben Stillstande gefübrt.

Aus dieser Uebersicht erhellt zur Genüge, dass unsere Kenntnisse rom diastolischen Stillstande noch ziemlich dürfig sind. Das ganze Kapitel hat durch die widersprechenden Befunde und die wonig plausiblen Erklärungsversucbe ein etwas mystisches Aussehen bekommen. Denn ist nun eine Hemmung durch Vagusreizung im Spiele oder nicht? Ist nicht die Zweischichtenhypothese, nag sie unsere Vorsteltungen noch so einfach gestalten, nicht sehr unwahrscheinlich, weil ihr alle histologische und physiologische Belege fehlen? Ist es in Werschinin's Erklärungsversuctron nicht unbegreiflich, dass bei böherer Konzentration die systolischen, bei niedrigerer die diastolischen Elemente zuerst vom resorbierten Gift erreicht werden? Und dass die Ringer'sche Lösung eine Kontraktion der Nahrungsspalten des Herzens verursachen würde, will uns obenso wenig einleuchten wie dio Angabe, dass sie innerbalb weniger Minuten die Muskel so undicht machon könnte, dass die Flüssigkeit vom Präparat abtropit.

Wir glauben, dass die Tatsachen eine einfachere Deutung zulassen, weun man nämlich bedenkt, dass der diastolische Stillstand eben keine besondere Abart des Herzstillstandes darstellt, sondero geradezu die „physiologische Art. Der systolische Stillstand dagegen ist etwas Aussergewöhnliches. Wenn die Bedingungen zum letzteren fehlen und eine Ursache zugegen ist, dio den Ventrikel zum Sistieren der Arbeit 
veranlasst, so ist der Stillstand ein diastolischer und die Erweiterung ist umsomehr ausgeprägt, je grösser der diastolische Füllungsdruck ist. Im Sinne Engolmann's kann der Stillstand begründot sein in negatip chronotroper, dromotroper, bathmotroper und inotroper Wirkung. Sowohl an Reizbildung als an Reizüberleitung als an Reizbarkeit der Kammer oder Leistongsfähigkeit,der Herzmuskel kañ̄ das Gift angreifen mit oder ohne Betoiligung des Vagusapparater. Von diesem Standpuntt aus soll man die Tatsachen zu deuten versuchen. Auch Winterberg (28) weist auf dies Moglichkeit bin. Der diastolisehe Kammerstillstand bei äusserliober Applikation, wie sie ron $J a c o b j$ und $W_{\text {y }}$ ba w beschrieben wurde, ist dann verursacht worden durch Störung der atrioventrikulären Reizäberleitung oder der Reizbarkeit der Kammer, denn der Vorbof setzte die Arbeit fort. Und es ist begreiflich, dass die Resorption in der winzig dännen Vorhof- and Sinuswand bei dieser Applikation rascher beendet ist als in dor dickeren Ventrikelwand, so dass der Ausdruck der Vergiftung letzterer, dor systolische Krampf, erst auftritt, wenn die Kammer infolge des Yangels an zufiessenden Reizen schon stillsteht. Wenn man mit Werschinin von innen her mit sehr dünnen Giftlösungen ebenfalls das Herz im disstolisohen Stillstande vorfindet, so mag dafür dieselbe Erklärung zutreffen. Allein hier tritt noch ein anderer Falstor ins Spiel, das ist die noptimale Belastung" von $20 \mathrm{om}$ Wasserdruck, unter der die Kammer am Wittiam'schen Apparat fortwahrend steht and die bewirken kann, dass am stillstehenden Herzen eine systolische Neigung, die an sich infolge der schwachen Giftkonzentration schon nicht gross ist, durch die passive Dehnung als Folge der hohen Wassersäule ganz maskiert wird. Auch wir haben bei unseren Versuchen mit höherem, z. B. $10 \mathrm{~mm}$ hohem venösem Druck öfters gesehen, wie ein am Ende der Vergiftung in halbsystolischer Stellung stillstehender Ventrikel innorhalb kurzer Zeit maximal systolisoh wurde, wenn der venöse Zufluss abgesperrt wurde.

Im Lichte unserer Anschauung erscheint der systolische Ventrikelstillstand Werschinin's nach innerlicher Vergiftung des isolierten Herzéns unverständlich. Donn varum würden jetzt von dieser höheren Konzentration dio Elemento in Vorhof- und Sinuswand geschont, die Muskelzellen elektiv vergiftet werden? Die Sache liegt jedoch anders. Der Gegensatz ist nicht so schroff. Erstens worden die Elemente in Vorhofsund Sinuswand nicht geschont: dem Stillstande geht eine Periode unregelmässiger Herzlätigkeit voraus. Es treten Störungen der Reizüberleitung auf, die man bei unserer Versucbsanordnung, im Gegensatz zur Williamschen, wo Vorhof und Sinus keine sichtliche Arboit leisten, besonders gut beobachten kann. Es fallen Systolen von Vorhof und Kammer oder von letzterer allein aus.

Zweitens ist der systolische Stillstand anfänglich kein eigentlicher Stillstand, sondern eine larvierte Pulsation. Die neue Muskelbeschaffenheit verhindert die diastolische Erweiterung. Wäre der Vorbof imstande, den Ventrikelkrampf zu überwinden, so würde die Kammer fortpulsieren. Und drittens findet man auch beim diastolischen Stillstande die Anzeichen der systolischen Wirkang, da Jacobj und Wybau beschreiben, dass das stillstehende Herz allmählich einen systolischen Stand annahm. 
Bei der Durchströmung des isolierten Herzens mit höheren Giftkonzentrationen spielt sich die ganze Vergiftung in raschem Tempo ab. Wę̧n die Reizüberleitung gesohädigt ist, ohne noch ganz zu versagen, ist es auch dem Herzmuskel gelungen, aus dem grossen Giftvorrat so viel zu schöpfen, dass die Kontraktur in die Erscheinung tritt. Am Williamschen Apparat genügt dann die noptimale Belastung " nicht mehr, diese systolische Neigung zu hompensieren. Der systolische Stillstand tritt ein.

Noch einen Umstand gibt es, don wir beim Entstehen des diastolischen Stillstandes beachten müssen, das ist die Alkalität der Flüssigkeit. Selbst haben wir im oben beschriebenen Versuch den schlagenden Effekt kleiner Reaktionsunterschiede auf den Stand des Ventrikels kennen gelernt. Wir sahen, dass eiwe zo niedrige Alkalität nach wenigen Pulsationen die Kammer diastolisch macht, dass dagegen eine za hohe Alkalităt einen systolischen Krampt erzeugt. Die Alkalitätsunterschiede nun scheinen uns zu die Erklärung abgeben zu müssen für die Beobachtung Werschinin's, dass dieselbe Giftkonzentration, die in Ringerscher Lösung diastolischen Stillstand macht, in derselben Lösung mit $1 / 9$ Blutserum das Herz systolisch arretiert. Denn aach für den Fall, dass der Autor für die Ringer'sche Lösung die optimale Alkalität gewäblt hat, was nicht feststeht, da er sich über die Reaktion der Nährflüssigkeiten in seiner Abhandlung nicht äussert, so bedeutet doch der Serumzusatz deshalb eine Verbesserung, weil im Serum ausser Bikarbonaten auch Phosphate und Eiweiss als Reaktionspuffer zagegen sind. Eine lokal durch die Muskelarbeit im Gewebe entstehendé saure Reaktion wird somit leichter abgestumpft.

Die Frage, ob der diastolische Stillstand bei äusserlicher Applikation einer Hemmung zuzuschreiben sei, wie Wybauw meint, lässt sich aus dem rorliegenden Tatsachenmaterial vicht ablehnend beantworten. Huldschinsky's Versuche vermögen keine Lösung herbeizufähren, denn es ist nicht recht einzusehen, weshalb eine an sich zum Stillstand ungenïgende elektrische Vagusreizung nicht eine durch das Helleborin unroittelbar geschädigto Reizbildung derart herabsetzen könnte, dass durch die Summierung beider Einflüsse der diastolische Stillstand eintritt, den 211 verursachen beide Faktoren an sich nicht ausreichten.

Auch die Folgerung Benedicenti's, dass die Hemmung ausgeschlossen ist, weil bei äusserlicher Vergiftung mit Strophanthin + Atropin der Stillstand doch diastolisch ist, ist nicht zwingend, denn die Möglicbkeit bleibt offen, dass dieser Stillstand von zweierlei Angriffspunten aus durch das Strophanthin erzwungen werden kapn: erstens am leichtesten, mittelbar, durch Wirkung auf den Vagasapparat, weloher Stillstand von Atropin vorgebeugt werden kánn, und zweitens, anmittolbar, durch direkto negativ chronotrope usw. Wirkang. Wenn man die Konzentration des Strophanthins, 1:25000, beobachtot, ist letzteres vicht un wahrscheinlich. Ein schwacher Punkt in Benedicenti's Versuchen-ist auch die bohe Atropinkonzentration: 1:50000. Das Atropin schwäcbt vach W y baur (24) die Herzmuskel und lassh dann die Erscheinung der Luciavi'schen Gruppen auftreten. Das Atropin könnte also in Benedicenti's Experimenten don diastolisohen Stillstand mit veranlasst haben. 
Ebensowenig lässt sich unseren Versuchon Material entaehmen, das die Entscheidung über die Beteiligung des Vagusapparates beim diastolischen Stillstande herbeiführen könnte. Sie wäre nachgewiesen, wonn kleine Atropingabén - etwa 1:1000000 - imstande wären, den Stillstand aufzuheben.

Kommen wir jetzt zur Besprechung der Art des Stillstandes in unseren Erythrophleinversuchen, so können wir uns darüber kurz lassen. Der Stillstand war'jn weitaus der Mohrzahl unserer Exporimente ein systolischer, fast ausnahmslos bei den höheren Konzentrationen: 1:200000, 1:300000, 1:500000. Bei den schwächeren: 1:1-2,5 Mill. fanden wir in weniger Fällen auch diastolischen Stillstand, wobei bald der Vorhof die Arbeit fortsetzte, bald jedoch aurh nicht. Ob in letzteren Versuchen ein Sinuaurikularblock vorlag, liess sich nicht immer entscheiden, da die Sinuspulsationen, wie schon oben gesagt warde, nicht inmer sichtbar waren.

Falls die Kammer diastolisch stiHstand, zeigte der Grad der Erweiterung noch erhoblicho Unterschiede bei den oinzelnen Versuchen. Sehr selten war der Stand oin maximal-diastolischer, meistens war dem rubenden Ventrikel der Anfang der systolischen Kontraktur doch schon anzusehen. Definitiv war der diastolische Stillstand in unseren Versuchen nie. Entweder wurdo-der Stillstand durchbrochen durch spontano Luciani'sche Reihen oder durch vereinzelte Systolen mit grösseren, z. B. halbminutenlangen Pausen. Im weiteren Verlaufo der Vergiftung kann man dann meistens beobachten, dass die Kammer in eine mehr systolische Stellung gerät. In einem Versuch stand 45 Min. nach dem Anfang der Vergiftung die Kammer still, der Vorbof folgte bald nach. 17 Minuten später war das ganze Herz noch diastolisch in Rube, fing jedoch sofort mit kräftigen Systolon lebhaft za pulsieren an, als dor Zuflussdruck akut gesteigert und unmittolbar wieder auf das alte Niveau zurückgebracht wurde. Nach einiger Zeif folgte ron neuem diastolischer Stillstand, der wieder auf dieselbe Weise unterbrochen wurde. Später traten spontan Systolenreihen auf, welche noch 40 Minuten nach dem Anfang des ersten Stillstandes erschienen, 2u welcher Zeit der Versuch abgebrochen wurde.

Noch eine Sache bleibt in bezug auf den diastolischen Stillstand zu erörtern übrig, das ist sein Zusammenhang mit der bekannten diastolischen Wirkung der Digitalissubstanzen, d. h. das Tieferwerden der diustolischen Erweiterung im Anfangsstadium der Vergiftung, eine Erscheinung, die zum Ansteigen des Schlagrolums beiträgt. Tritt diese zugleich auf mit einem Herabsinken der Pulszahl, so braucht sie nichts für einen spezifischen Einfluss auf die Elastizität der Herzmuskel zu beweisen. Wohl hat sie dafür Bedeutung, wenn die Frequenz sich nicht ändert [cf. Winterberg (28)]. Auch wir haben eine derartige spezifische diastolische Wirkung in einigen Fällen konstatieren können und zwar hauptsächlich im Initialstadium. Erholte sich das Herz aus diesem, so wurde die systolische Zusammenziehung fortwährend vollständiger, die diastolische Erweiterung jedoch blieb anfänglich ausyiebig. Die Ventrikel- 
exkursion war also nach beiden Seiten hin erweitert worden. Zeitlich liegen aber diese diastolische Wirkung und der diastolische Stillstand weit auseinander und es ist nicht etwa so, dass im Verlaufe der Vergiftung die diastolische Wirkung immerfort stärker sich ausprägt, um im diastolischen Stillstande zu enden. Sie bleibt vielmehr bis zum Stillstand auf einer selben Höhe, wenn wenigstens nicht vor diesef Zeit der An. fang der tonischen Kontraktur in die Erscheinung getreten ist.

Wir sind denin auch der Meinung zugetan, dass der diastolische Stillstand in ganz anderen, oben umschriebenen Ursachen begründet ist, als die spezifische diastolische Wirkung auf die Herzmuskel.

Aber die rielgeliebte Ausdrucksweise: "Wie der systolische Stillstand das Eude der systolischen Wirkung, so ist auch der diastolische Stillstand das Ende der diastolischen " ist nach unserer Meinung auch im ersten Teil nicht richtig. Bei der Besprechung der Elastizitätsänderungen sind wir zum Schluss gekommen, dass die systolische Erythrophleinkontraktur auf anderen Grundlagen fassen muss als die eigentliche Kontraktion. Nun ist die,systolische Wirkung nichts anderes als eine Verbesserung der Zusammenziehung der Kammer. Erschlafft diese, so kommt sio im ersten Stadium der Vergiftung in der Pause in eine Stellung, die mehr diastolisch ist als vor der Vergiftung, was sich mit einer tonischen Kontraktur nicht zusammenreimen lässt. Systolische Wirkung und systolischer Stillstand-sind also ebenfalls als getrennte Wirkungen des Giftes aufzufassen.

\section{Zasammenfassnng.}

In 49 Versuchen studierten wir die Wirkung des Erythrophleins auf des isolierte Herz von Rana temporaria. Dabei stellte sich, heraus, dass die Sabstanz die Grundcharaktere der Digitaliswirkung hervortreten lässt:

1. Das Erytbrophlein verursacht fast regelmässig eine Pulsverlangsamung, die schon in einem frühen Stadium der Vergiftung eintritt. In einem Versuch sahen wir, dass durch Atropin ein Teil der darch das Erythrophlein rerursachten Pulsverlangsamung wieder aufgehoben wurde. Wir vermuten daher, dass diese Eirgthrophleinwirkung nicht nur an den automatischen Zentren selbst, sondern auch an den intrakardialen Vaguselementen angreift.

2. Auch unabhängig von dieser Frequenzverminderung wird die Diastole vertieft. Oft war dicse Vertiefung der Rest der starken ninitialen" Erweiterung.

3. Die Systole des Ventrikels nimmt an Energie zu, die Zusammenziehung ist eine vollständigere als vor der Vérgiftung. Falls die Frequenz nicht zuviel abnimmt, folgt hieraus eine Steigerung des Minutvolums.

4. Bei genügender Konzentration tritt die bekannte tonische Kontraktur auf, die sich anfänglich in einer Erschwerang der diastolischen Erweiterung äussert. Das Ende ist der systolische Stillstand. .Der Vorhof kann die Arbeit noch einige Zeit fortsetzen. Bein Entstehen dieser Kontraktur konnten wir einige Male das „Elastizitätsphänomen“ " beobachten. Wir sind der Ansicht, dass die systolische Kontraktur und die 
Verbesseruug der Systole in $\mathbf{2 w e i}$ verschiedenen Wirtungen des Giftes begründet sind.

5. Einen diastoljschen Stillstand sahen wir in einer kleinen Anzahl unserer $\nabla$ ersuche bei scbwachen Konzentrationen des Giftes. Er war vie definitiv.

Dieser Stillstand berubt nicht auf einer besonderen diastolischen Wirkang des Giftes anf den Horzmuskel, sondern wird verursacht durch negativ chronotrope, dromotrope, bathmotrope und vielleicht inotrope Wirkungen des Giftes auf das Herz, dessen Muskel nooh nicht so weit vergiftet ist, dass die tonische Kontraktur in dio Erscheinung trin. Es stebt der diastolische Stillstand also in keinem dirotten Zusammonhang mit der die Diastole vertiofende Wirkung des Girtes im Anfang der Vergiftung.

6. Die Reizloitung (oder die Reizbarkeit) wird rom Erythrophlein in negativer Weise beeinfusst. Besonders das geschädigte Reizleitungssystem ist für das Gift sehr empfindlich. Einmal haben wir bei eiver Rana esculenta eine Steigerung der Automatio des Ventrikels unter der Form einer atrioventrikuläron Dissoziation wahrnehmen können.

7. In otwa der Hälfte unserer-Versuche (auch diejenigen mitgerechnet, wo es nur andeufungswoise auftrat) haben wir im Anfang der Vergiftung einen Komplex von Erscheinungen wahrnehmen könnon, die wir das "Initialstadium* genannt haben. Der Hauptsache nach bestand es aus einem Diastolischwerden der Kammer, sobald das Gift dio Herzmuskel erreicht hatte. Hierbei sanken Minutrolum, Minutarbeit, Pulsvolum, Pulsarbcit und öfters auch die Frequenz herab. Wenn diese letztere nicht zu stark herabsank, war eine Kiederherstellung dur Funktion die Regel. Infolge der übrigbleibenden Vertiefung der Diastole und der jetzt auftretenden Verbesserang der Systole kam diese Erbolung oft über das Nireau der Herzleistung por der Vergiftung hinans. Zur Erklärung des Entstehens dieses Initialstadiums meinen wir, da wir es offenbar mit einer Kontaktwirkung und keiner Rosorptionswirkung zu tun haben, die Endokardnerven berücksichtigen zu müssen.

Auf die Auhnlichkeit des Initialstadiums mit èiner Vagusreizung, speziell mit der Muskarinvergiftung, haben wir hingewiesen.

8. Von grossem Einfluss auf die Herzwirkung ist der Alkalitätsgrad der Durchströmungsfiüssigkeit. Ein sehr kleiner Unterschied in der Alkalität genügt, allo Funktionen der Herzwirkung in eklatanter Weise herabzudrücken oder zu verstärzen.

\section{Literaturverzeichnis.}

1) Ginsberg und Hoblberg, Zur Frage der Standardisation (Normioren) von Horzmittoln. 11. Intern. Kongr. f. Pharmazio. S. 66. - 2) Quillemin, Perrotbe ot Ric bard, Florae Sonegambia tentamen. Parisiis 1830. I. p. 242. (Gute Abbildungen ron Blüten, Blättern nod Fräobten.) - 3) Gallo is et Hardy, Soo. do biol. 1876. - 4) Brunton and Pyde, On the action of casca bark. Bartholom. bosp. rop. 1876. Vol. XII. p. 125. Phil, trans. of the Roy. soc. 1876. - 5) S6́ et Bochofontaine, Sur les effots physiologiques de l'érythrophlëine. Compt. rend. 1880. T. 1. 
p. 1366. - 6) Lewin, Berl. klin. Worhonschr. 1888. S. 61 a. 134. Liobroich, Berl. klin. Wochenschr. 1888. S. 161 a. 190 . Kollor, Wienor mod. Wochensohr. 1888. S. 186. Kaposi, Wiener mod. Wochenscbr. 1888. S. 282. Lipp, Wiener mod. Wochenschr. 1888. S. 354. Loowonbardt, Berl. Hlin. Wochenschr. 1888. S. 189. - 7) Hermann, Ueber die Wirkung dos Erytbrophleins auf das Herz. Wiener klin. Wochensobr. 1888. S. 197. - 8) S60, J'érytbrophlaino, médicament oargiaque. Somaine mód. 1891. No. 49. - 9) Drammoud, Cassa bart rersus digitalis. Tho Lancet. 1880. Il. p. 763. - 10) Harna ok and Zaturooki, Untersuchengen über das Erythrophlein, don wirksamen Bestandteil der Sassyrinde. Arch. f. exp. Pathol. a. Pharmakol. 1882. Bd. 15. S. 403. - 11) Harna ok, Chemisoh.pharmakologischo Untersuchangen über das Erytbrophlein. $\Delta$ rch. d. Pharmazio. 1896. Bd. 234. S. 561. - 12) Jacobj, Zar Physiologio des Horzeng untor Beräcksichtigang dor Digitalis. wirkuog. Arch. f. expor. Patbol. u. Pbarm. 1900. Bd. 44. S. 368. - 13) Jacobj and Roomor, Ueber Veronal. Arch. f. exper. Patb. u. Pbarm. 1911. Bd.66. S. 275. - 14) Baroroft, The respiratory function of the blood. Cambridge 1914. - 15) Straub, Beckmann, Erdt and Mettenloiter, Alreolargasanalysen. Deutsohes Arcb. f. klin. Mod. 1915. Bd. 117. S. 397. - 16) Traube, Gesamneite Beiträge zur Physiologio und Pathologio. 1871. - 17) Rotbberger und Winterberg, Uober den Finfluss ron Stropbanthin auf dio Reizbildungsfähigheit dòr automatischon Zentron des Herzens. Pfluger's Arch. 1913. Bd. 150. S. 217. - 18) Hofmann, Nagel's Handb. d. Phys. I. S. 246. - 19) Suiroow, Heijmans and Domoor, Schmidt, zit. nacb Wybauw. - 20) Wybaum, Beitrag zur Kenntnis der pharmakologiscbon Wirkung der Stoffe aus der Digitalisgruppo. Arch. f. exper. Path. u. Pharm. 1900. Bd. 44. S. 434. - 21) Cashny, On the action of substances of the digitalis series on the oirculation in mammals. Journ. of oxp. med. 189i. Vol. II. p. 233. - 22). de Boor, Bijdrage tot do kennis van de werking ran digitalis op het kikkerbart. Spontane en experimenteele rbythmewisselingen. Nederl. T'ijdschr. P. Geneesk. 1917. p. 701. - 23) Jacobj, 1. c. - 24) Wybauw, 1. o. - 25) Haldschinskg, Ueber de berzhemmende Digitalinwirkung. Arch. f. exper. Patb. a. Pharm. 1908. Bd. 58. S. 413. - 26) Benediconti, Ueber die Wirkung der Stofie der Digitalingruppe bei exokardialer Applikation. Arch. f. exper. Patb. u. Pbarm. 1903. Bd. 47. S. 360. 27) Worsolinin, Zur konntnis der diastolischen Horzwirkung der Digitalingruppe. Arch. f. exper. Path. a. Pharm. 1909. Bd. 60. S. 328. 1910. Bd. 63. S. $386 .-$ 28) Winterberg, in v. Jagic's Handb. d. Herz- u. Gefasserkrank. Bd. II. S. 603. 